Pacific Journal of Mathematics

SUFFICIENCY AND RELATIVE ENTROPY IN *-ALGEBRA
WITH APPLICATIONS IN QUANTUM SYSTEMS 


\title{
SUFFICIENCY AND RELATIVE ENTROPY IN *-ALGEBRAS WITH APPLICATIONS IN QUANTUM SYSTEMS
}

\author{
Fumio Hiai, Masanori OHya AND MaKoto TsuKada
}

The sufficiency and weak sufficiency in *-algebras are discussed. Some properties are studied concerning the relative entropy and the sufficiency for invariant states and KMS states in $W^{*}$ - and $C^{*}$-dynamical systems.

Introduction. The concept of sufficiency is very important in mathematical statistics. The abstract measure theoretic investigation of sufficient statistics was initiated by Halmos and Savage [13]. Kullback and Leibler [19] gave the characterization of sufficiency in terms of the information (i.e., the classical relative entropy). Umegaki $[33,34]$ studied the sufficiency and the relative entropy in the noncommutative case of semi-finite von Neumann algebras.

Araki $[4,5]$ extended the relative entropy to the case for normal positive linear functionals of general von Neumann algebras and showed its several properties. Furthermore Uhlmann [32] showed the general WYDL concavity using a quadratic interpolation theory and defined the relative entropy of positive linear functionals of arbitrary $*$-algebras.

In the previous paper [14], we discussed the sufficiency and the relative entropy in von Neumann algebras and gave the characterizations of invariant states and KMS states with respect to the modular automorphism group of a faithful normal state.

In this paper, we further develop the sufficiency and the relative entropy in $*$-algebras. In $\S 1$, we introduce besides the sufficiency another notion of weak sufficiency and establish the relation between them. In $\S 2$, we deal with the weak sufficiency of positive linear maps between $*$-algebras. In $\$ 3$, we mention the Araki's and Uhlmann's relative entropies which are equal in the von Neumann algebra case. We further give a formula of relative entropy for states of $C^{*}$-algebras. In $\S 4$, we establish some properties of invariant states and KMS states in $W^{*}$-dynamical systems and $C^{*}$-dynamical systems through the relative entropy and the sufficiency. The theorems there improve or extend the results obtained in [14]. Finally we give an application to the Gibbs states of quantum lattice systems. 
The authors wish to express their gratitude to Professor H. Umegaki for his advice and encouragement.

1. Sufficiency and weak sufficiency of $*$-subalgebras. In this paper, we shall assume that all $*$-algebras, $C^{*}$-algebras and von Neumann algebras have the unity $I$ and their $*$-subalgebras always contain $I$. Let $\mathbb{Q}$ be a $*$-algebra and $\mathcal{S}$ be the set of all states of $\mathcal{Q}$.

Definition 1.1. A *-subalgebra $\mathscr{B}$ of $\mathscr{Q}$ is said to be sufficient for $S \subset \mathcal{S}$ if there exists a projection $\varepsilon$ of $\mathbb{Q}$ onto $\mathscr{Q}$ such that

(i) $\varepsilon\left(A^{*}\right)=\varepsilon(A)^{*}$ for all $A \in \mathbb{Q}$,

(ii) $\varepsilon(A)^{*} \varepsilon(A) \leq \varepsilon\left(A^{*} A\right)$ for all $A \in \mathbb{Q}$,

(iii) $\varepsilon\left(B_{1} A B_{2}\right)=B_{1} \varepsilon(A) B_{2}$ for all $A \in \mathbb{Q}$ and $B_{1}, B_{2} \in \mathscr{P}$,

(iv) $\varphi=\varphi \circ \varepsilon$ for all $\varphi \in S$.

We here call a projection $\varepsilon$ of $\mathscr{Q}$ onto $\mathscr{B}$ satisfying (i)-(iii) a conditional expectation of $\mathscr{Q}$ onto $\mathscr{B}$. If $\mathscr{Q}$ is a $C^{*}$-algebra and $\mathscr{B}$ is a $C^{*}$-subalgebra, then a conditional expectation of $\mathscr{Q}$ onto $\mathscr{Q}$ is nothing but a norm one projection of $\mathbb{Q}$ onto $\Re$ (cf. [31]).

We first give some examples of sufficiency in von Neumann algebras. Let $\mathfrak{R}$ be a von Neumann algebra and $\subseteq$ be the set of all normal states of $\mathfrak{n}$. The definition in [14] of sufficiency of a von Neumann subalgebra for $S \subset \subseteq$ is somewhat different from Definition 1.1. However these are equivalent if $S$ contains a faithful normal state (this is the case dealt in [14]).

EXAMPLE 1.2. Let $\varphi \in \subseteq$ be faithful and $\sigma_{t}^{\varphi}$ be its modular automorphism group (cf. [28]). We showed in [14] that the centralizer of $Z_{\varphi}$ of $\varphi$ is sufficient for the set of all $\sigma_{t}^{\varphi}$-invariant states in $\subseteq$ and the center $3=\mathfrak{R} \cap \mathfrak{R}^{\prime}$ is sufficient for the set of all states in $\subseteq$ satisfying the KMS condition with respect to $\sigma_{t}^{\varphi}($ at $\beta=1)$.

EXAMPLE 1.3. Assume that $\mathfrak{N}$ is semi-finite with a faithful normal semi-finite trace $\tau$ of $\mathfrak{A}$. For each $\varphi \in \mathcal{S}$, there exists a unique positive self-adjoint operator $\rho_{\varphi}=d \varphi / d \tau$ such that $\varphi(A)=\tau\left(\rho_{\varphi} A\right)$ for all $A \in \mathfrak{R}$. For any set $S \subset \subseteq$, the von Neumann subalgebra $\mathfrak{M}$ generated by $\{d \varphi / d \tau: \varphi \in S\}$ is proved to be sufficient for $S$ (see [16, p. 72]).

EXAMPLE 1.4. Let $\{\Re, G, \alpha\}$ be a $W^{*}$-dynamical system where $g \mapsto \alpha_{g}$ is a representation of a group $G$ in $\operatorname{Aut}(\mathfrak{R})$. Let $\mathfrak{R}^{\alpha}$ be the fixed point subalgebra of $\alpha$ and $\varsigma_{\alpha}$ be the set of all $\alpha$-invariant states in $\subseteq$. Then the 
result of Kovács and Szücs [18] asserts that if $\mathfrak{N}$ is $G$-finite, i.e., $\varphi\left(A^{*} A\right)=0$ for all $\varphi \in \subseteq_{\alpha}$ implies $A=0$, then $\mathfrak{N}^{\alpha}$ is sufficient for $\mathfrak{S}_{\alpha}$.

For $*$-subalgebras $\mathscr{B}$ of $\mathcal{Q}$, the existence of a conditional expectation of $\mathscr{Q}$ onto $\mathscr{B}$ is usually a rather strict condition. In the sequel, we introduce another weak notion of sufficiency by using cyclic representations of $Q$. Unbounded $*$-representations of $*$-algebras were studied in [23]. A $*$-representation $\pi$ of $\mathcal{Q}$ on a Hilbert space $\mathcal{H}$ is a map of $\mathcal{Q}$ into linear operators all defined on a common dense domain $D(\pi) \subset \mathcal{H}$ which satisfies $\pi(I)=I$ and

(i) $\pi(\alpha A+\beta B) \Phi=\alpha \pi(A) \Phi+\beta \pi(B) \Phi$ for all $A, B \in \mathbb{Q}, \alpha, \beta \in \mathbf{C}$ and $\Phi \in D(\pi)$,

(ii) $\pi(A) D(\pi) \subset D(\pi)$ for all $A \in \mathbb{Q}$ and $\pi(A) \pi(B) \Phi=\pi(A B) \Phi$ for all $A, B \in Q$ and $\Phi \in D(\pi)$,

(iii) $\langle\Phi, \pi(A) \Psi\rangle=\left\langle\pi\left(A^{*}\right) \Phi, \Psi\right\rangle$ for all $\Phi, \Psi \in D(\pi)$, i.e., $\pi\left(A^{*}\right) \subset$ $\pi(A)^{*}$ for all $A \in \mathbb{Q}$.

The unbounded commutant $\pi(\mathbb{Q})^{c}$ of $\pi(\mathbb{Q})$ consists of all linear operators $T: D(\pi) \rightarrow \mathcal{H C}$ such that

$$
\langle\Phi, T \pi(A) \Psi\rangle=\left\langle\pi\left(A^{*}\right) \Phi, T \Psi\right\rangle, \quad A \in \mathbb{Q}, \Phi, \Psi \in D(\pi) .
$$

The commutant $\pi(\mathscr{Q})^{\prime}$ of $\pi(\mathscr{Q})$ is the set of all bounded operators $T$ on $\mathcal{H}$ such that $T \backslash D(\pi) \in \pi(\mathbb{Q})^{c}$. For each positive linear functional $\varphi$ of $\mathscr{Q}$, the GNS construction gives rise to a cyclic representation $\left\{\mathcal{H}_{\varphi}, \pi_{\varphi}, \Omega_{\varphi}\right\}$ of $\mathcal{Q}$ induced by $\varphi$ which is unique up to unitary equivalence, that is, $\pi_{\varphi}$ is a *-representation of $\mathscr{Q}$ on a Hilbert space $\mathcal{H}_{\varphi}$ with $\Omega_{\varphi} \in D\left(\pi_{\varphi}\right)$ such that

$$
\begin{gathered}
D\left(\pi_{\varphi}\right)=\pi_{\varphi}(\mathscr{Q}) \Omega_{\varphi}, \quad \mathcal{H}_{\varphi}=\overline{\pi_{\varphi}(\mathscr{Q}) \Omega_{\varphi}}, \\
\varphi(A)=\left\langle\Omega_{\varphi}, \pi_{\varphi}(A) \Omega_{\varphi}\right\rangle, \quad A \in \mathbb{Q} .
\end{gathered}
$$

If for every $A \in \mathbb{Q}$ there exists a $c>0$ with $A^{*} A \leq c I$ (particularly if $\mathscr{Q}$ is a $C^{*}$-algebra), then $\pi_{\varphi}$ becomes a bounded $*$-representation of $\mathscr{Q}$ on $\mathcal{H}_{\varphi}$. We shall use in this paper the following three conditions of absolute continuity.

(1) A positive linear functional $\psi$ is absolutely continuous with respect to $\varphi$ (we write $\psi \ll \varphi$ ) if $\varphi\left(A^{*} A\right)=0$ implies $\psi\left(A^{*} A\right)=0$.

(2) A linear functional $\psi$ is strongly absolutely continuous with respect to $\varphi$ (we write $\psi \prec \varphi$ ) if for each sequence $\left\{A_{n}\right\}$ in $Q, \varphi\left(A_{n}^{*} A_{n}\right) \rightarrow 0$ implies $\psi\left(B A_{n}\right) \rightarrow 0$ for all $B \in \mathbb{Q}$.

(3) A positive linear functional $\psi$ is dominated by $\varphi$ if $\psi \leq c \varphi$ for some $c>0$.

Note that for any positive $\psi,(3)$ implies (2) and (2) implies (1). If $\psi$ is a linear functional of $\mathbb{Q}$ with $\psi \prec \varphi$, then by [11, Theorem 1] there exists a 
unique $T \in \pi_{\varphi}(\mathbb{Q})^{c}$ (we denote by $T=d \psi / d \varphi$ ) such that

$$
\psi(A)=\left\langle T \Omega_{\varphi}, \pi_{\varphi}(A) \Omega_{\varphi}\right\rangle, \quad A \in \mathbb{Q} .
$$

Then $\psi$ is positive if and only if $T$ is positive, and moreover $\psi$ is dominated by $\varphi$ if and only if $T$ is bounded so that $T \in \pi_{\varphi}(\mathbb{Q})^{\prime}$. For each *-subalgebra $\mathscr{\Re}$ of $\mathscr{Q}$, let $\Re_{\varphi}=\pi_{\varphi}(\Re) \Omega_{\varphi}$ and $\overline{\Re_{\varphi}}=\overline{\pi_{\varphi}(\Re) \Omega_{\varphi}}$. For every $A \in \mathbb{Q}$, we define a vector $P_{\varphi}(A \mid \mathscr{B})$ in $\overline{\mathscr{B}_{\varphi}}$ by

$$
P_{\varphi}(A \mid \Re)=P_{\overline{\mathscr{B}_{\varphi}}}\left(\pi_{\varphi}(A) \Omega_{\varphi}\right)
$$

where $P_{\overline{\mathscr{B}_{\varphi}}}$ is the orthogonal projection onto $\overline{\mathscr{B}_{\varphi}}$.

Definition 1.5. A *-subalgebra $\Re$ of $Q$ is said to be weakly sufficient for $S \subset \mathcal{S}$ if for each $A \in \mathbb{Q}$ there exists a sequence $\left\{B_{n}\right\}$ in $\Im$ such that

$$
P_{\varphi}(A \mid \Re)=s-\lim \pi_{\varphi}\left(B_{n}\right) \Omega_{\varphi}, \quad \varphi \in S .
$$

THEOREM 1.6. Assume that there is a finite subset $\left\{\varphi_{1}, \ldots, \varphi_{k}\right\}$ of $S$ such that every $\varphi \in S$ is dominated by $\rho=\sum_{l=1}^{k} \varphi_{l}$. Then a $*$-subalgebra $\Re$ of $Q$ is weakly sufficient for $S$ if and only if $(d \varphi / d \rho) \Re_{\rho} \subset \overline{\Re_{\rho}}$ for every $\varphi \in S$.

Proof. Suppose that $\mathscr{B}$ is weakly sufficient for $S$. For each $A \in \mathbb{Q}$, there exists a sequence $\left\{B_{n}\right\}$ in $\mathscr{B}$ such that

$$
P_{\varphi}(A \mid \Re)=s-\lim \pi_{\varphi}\left(B_{n}\right) \Omega_{\varphi}, \quad \varphi \in S .
$$

Since $\left\{\pi_{\rho}\left(B_{n}\right) \Omega_{\rho}\right\}$ is Cauchy, it follows that $\Psi=s-\lim \pi_{\rho}\left(B_{n}\right) \Omega_{\rho}$ exists in $\overline{\mathscr{G}_{0}}$. If $B \in \Re$, then we have

$$
\begin{aligned}
\left\|\pi_{\rho}(A) \Omega_{\rho}-\pi_{\rho}(B) \Omega_{\rho}\right\|^{2} & =\sum_{l=1}^{k}\left\|\pi_{\varphi_{l}}(A) \Omega_{\varphi_{l}}-\pi_{\varphi_{l}}(B) \Omega_{\varphi_{l}}\right\|^{2} \\
& \geq \sum_{i=1}^{k}\left\|\pi_{\varphi_{l}}(A) \Omega_{\varphi_{t}}-P_{\varphi_{t}}(A \mid \Re)\right\|^{2} \\
& =\lim \sum_{l=1}^{k}\left\|\pi_{\varphi_{l}}(A) \Omega_{\varphi_{l}}-\pi_{\varphi_{l}}\left(B_{n}\right) \Omega_{\varphi_{l}}\right\|^{2} \\
& =\lim \left\|\pi_{\rho}(A) \Omega_{\rho}-\pi_{\rho}\left(B_{n}\right) \Omega_{\rho}\right\|^{2} \\
& =\left\|\pi_{\rho}(A) \Omega_{\rho}-\Psi\right\|^{2},
\end{aligned}
$$

so that $P_{\rho}(A \mid \Re)=\Psi=s-\lim \pi_{\rho}\left(B_{n}\right) \Omega_{\rho}$. For each $\varphi \in S$, let $T=d \varphi / d \rho$ and $\hat{T}=d(\varphi \mid \Re) / d(\rho \mid \Re)$ where the cyclic representation of $\Re$ induced 
by $\rho \uparrow \Re$ is given by $\left\{\overline{\mathscr{G}_{\rho}}, \pi_{\rho} \uparrow \Re, \Omega_{\rho}\right\}$. Then for every $B \in \mathscr{B}$ we have

$$
\begin{aligned}
\left\langle T \pi_{\rho}(B) \Omega_{\rho}, \pi_{\rho}(A) \Omega_{\rho}\right\rangle & =\varphi\left(B^{*} A\right)=\left\langle\pi_{\varphi}(B) \Omega_{\varphi}, P_{\varphi}(A \mid \Re)\right\rangle \\
& =\lim \varphi\left(B^{*} B_{n}\right)=\left\langle\hat{T} \pi_{\rho}(B) \Omega_{\rho}, P_{\rho}(A \mid \Re)\right\rangle \\
& =\left\langle\hat{T} \pi_{\rho}(B) \Omega_{\rho}, \pi_{\rho}(A) \Omega_{\rho}\right\rangle .
\end{aligned}
$$

Since this holds for each $A \in \mathbb{Q}$, we obtain

$$
T \pi_{\rho}(B) \Omega_{\rho}=\hat{T} \pi_{\rho}(B) \Omega_{\rho} \in \overline{\mathscr{B}_{\rho}}, \quad B \in \Re,
$$

and hence $T \mathscr{B}_{\rho} \subset \overline{\mathscr{G}_{\rho}}$.

Conversely suppose that $(d \varphi / d \rho) \mathscr{\Re}_{\rho} \subset \overline{\mathscr{B}_{\rho}}$ for all $\varphi \in S$. Let $A \in \mathbb{Q}$ and take a sequence $\left\{B_{n}\right\}$ in $\Re$ such that $P_{\rho}(A \mid \Re)=s-\lim \pi_{\rho}\left(B_{n}\right) \Omega_{\rho}$. For each $\varphi \in S$, since $\varphi$ is dominated by $\rho$, it follows that $\left\{\pi_{\varphi}\left(B_{n}\right) \Omega_{\varphi}\right\}$ is Cauchy, so that $\Phi=s-\lim \pi_{\varphi}\left(B_{n}\right) \Omega_{\varphi}$ exists in $\overline{\mathscr{B}_{\varphi}}$. If $B \in \Re$, then we have

$$
\begin{aligned}
\left\langle\pi_{\varphi}(B) \Omega_{\varphi}, P_{\varphi}(A \mid \Re)\right\rangle & =\varphi\left(B^{*} A\right)=\left\langle(d \varphi / d \rho) \pi_{\rho}(B) \Omega_{\rho}, P_{\rho}(A \mid \Re)\right\rangle \\
& =\lim \varphi\left(B^{*} B_{n}\right)=\left\langle\pi_{\varphi}(B) \Omega_{\varphi}, \Phi\right\rangle,
\end{aligned}
$$

and hence $P_{\varphi}(A \mid \Re)=\Phi=s-\lim \pi_{\varphi}\left(B_{n}\right) \Omega_{\varphi}$. Thus $\Re$ is weakly sufficient for $S$.

REMARK. Theorem 1.6 is considered as the noncommutative extension of Halmos-Savage's theorem [13]. For the proof of "only if" part of Theorem 1.6, we need only $\varphi \prec \rho$ for every $\varphi \in S$. If $\pi_{\rho}$ is a bounded *-representation (particularly if $\mathscr{Q}$ is a $C^{*}$-algebra), we see that $(d \varphi / d \rho) \Re_{\rho}$ $\subset \overline{\mathscr{G}_{\rho}}$ is equivalent to $(d \varphi / d \rho) \Omega_{\rho} \in \overline{\mathscr{G}_{\rho}}$ since $T \pi_{\rho}(A) \Omega_{\rho}=\pi_{\rho}(A) T \Omega_{\rho}$ for all $A \in \mathbb{Q}$ and $T \in \pi_{\rho}(\mathbb{Q})^{c}$.

In the following theorem, we state the elementary facts of weak sufficiency which are immediately seen from the definition and Theorem 1.6.

THEOREM 1.7. (1) If a *-subalgebra $\Re$ of $\mathbb{Q}$ is weakly sufficient for $\{\varphi, \psi\}$ and $\varphi=\psi$ on $\Re$, then $\varphi=\psi$ on $Q$.

When the assumption in Theorem 1.6 is satisfied, then:

(2) If a *-subalgebra $\Re$ of $Q$ is weakly sufficient for $S$, then $\Re$ is weakly sufficient for the convex hull of $S$.

(3) If a *-subalgebra $\Re$ of $Q$ is weakly sufficient for $S$ and a $*$-subalgebra $\mathcal{C}_{\text {of }} \Re$ is weakly sufficient for $\{\varphi \uparrow \Re: \varphi \in S\}$, then $\mathcal{C}_{\text {is }}$ weakly sufficient for $S$.

(4) If a *-subalgebra $\Re$ of a $C^{*}$-algebra $Q$ is weakly sufficient for $S$, then any *-subalgebra $\mathcal{C}$ with $\Re \subset \subset \subset \notin$ is weakly sufficient for $S$. 


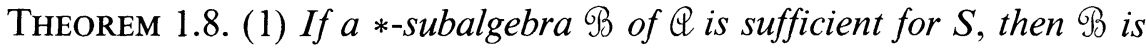
weakly sufficient for $S$.

(2) Assume that there is a $\varphi \in S$ such that $\psi \prec \varphi$ for all $\psi \in S$. Then a *-subalgebra $\Re$ of $Q$ is sufficient for $S$ if and only if $\Re$ is weakly sufficient for $S$ and there exists a conditional expectation $\varepsilon_{\varphi}$ of $\Theta$ onto $\Re$ with $\varphi=\varphi \circ \varepsilon_{\varphi}$.

Proof. (1) Let $\varepsilon$ be a conditional expectation of $\mathscr{Q}$ onto $\mathscr{B}$ with $\varphi=\varphi \circ \varepsilon$ for all $\varphi \in S$. If $A \in \mathbb{Q}, B \in \mathscr{B}$ and $\varphi \in S$, then we have

$$
\begin{aligned}
\left\langle P_{\varphi}(A \mid \Re), \pi_{\varphi}(B) \Omega_{\varphi}\right\rangle & =\varphi\left(A^{*} B\right)=\varphi(\varepsilon(A) * B) \\
& =\left\langle\pi_{\varphi}(\varepsilon(A)) \Omega_{\varphi}, \pi_{\varphi}(B) \Omega_{\varphi}\right\rangle,
\end{aligned}
$$

and hence $P_{\varphi}(A \mid \mathscr{B})=\pi_{\varphi}(\varepsilon(A)) \Omega_{\varphi}$. Thus $\mathscr{B}$ is weakly sufficient for $S$.

(2) Suppose that $\mathscr{B}$ is weakly sufficient for $S$ and there exists a conditional expectation $\varepsilon_{\varphi}$ of $\mathbb{Q}$ onto $\Re$ with $\varphi=\varphi \circ \varepsilon_{\varphi}$. We show that $\psi=\psi \circ \varepsilon_{\varphi}$ for all $\psi \in S$. For each $\psi \in S$, since $(d \psi / d \varphi) \Omega_{\varphi} \in \overline{\mathscr{G}_{\varphi}}$ by Theorem 1.6 (Remark), we can choose $\left\{B_{n}\right\}$ in $\mathscr{B}$ such that

$$
(d \psi / d \varphi) \Omega_{\varphi}=s-\lim \pi_{\varphi}\left(B_{n}\right) \Omega_{\varphi} .
$$

Then $\psi=\psi \circ \varepsilon_{\varphi}$ follows from

$$
\begin{aligned}
& \psi\left(\varepsilon_{\varphi}(A)\right)=\left\langle(d \psi / d \varphi) \Omega_{\varphi}, \pi_{\varphi}\left(\varepsilon_{\varphi}(A)\right) \Omega_{\varphi}\right\rangle \\
& \quad=\lim \left\langle\pi_{\varphi}\left(B_{n}\right) \Omega_{\varphi}, \pi_{\varphi}\left(\varepsilon_{\varphi}(A)\right) \Omega_{\varphi}\right\rangle=\lim \varphi\left(B_{n}^{*} \varepsilon_{\varphi}(A)\right) \\
& \quad=\lim \varphi\left(B_{n}^{*} A\right)=\left\langle(d \psi / d \varphi) \Omega_{\varphi}, \pi_{\varphi}(A) \Omega_{\varphi}\right\rangle=\psi(A), \quad A \in \mathbb{Q} .
\end{aligned}
$$

EXAMPLE 1.9. We recall the usual concept of sufficiency in the classical probability theory $(\mathrm{cf} .[7,13])$. Let $(X, \mathscr{F})$ be a measurable space and $S$ be a set of probability measures on $\mathscr{F}$. A $\sigma$-subalgebra $\mathcal{G}$ of $\mathscr{F}$ is sufficient for $S$ if and only if for each $A \in \mathscr{F}$ there exists a $\mathcal{G}$-measurable function $g$ such that $g=E_{\mu}\left(1_{A} \mid \mathcal{G}\right)$ a.e. $[\mu]$ for every $\mu \in S$, where $E_{\mu}\left(1_{A} \mid \mathcal{G}\right)$ denotes the conditional expectation of the characteristic function $1_{A}$ of $A$ with respect to $\mu$ and $\mathcal{G}$. Let $\mathscr{Q}$ (resp. $\mathscr{B}$ ) be the set of all complex-valued $\mathscr{F}($ resp. $\mathcal{G})$-measurable simple functions. Under the pointwise operations, $\mathbb{Q}$ becomes a $*$-algebra and $\Re$ is a $*$-subalgebra of $\mathbb{Q}$. Each $\mu \in S$ is naturally regarded as a state of $\mathcal{Q}$. The cyclic representation $\left\{\mathcal{H}_{\mu}, \pi_{\mu}, \Omega_{\mu}\right\}$ is given as follows: $\mathcal{H}_{\mu}=L^{2}(X, \mathcal{F}, \mu), \pi_{\mu}(f)$ is the multiplication operator by $f \in \mathbb{Q}$, and $\Omega_{\mu}=1$. Moreover $\overline{\mathfrak{B}_{\mu}}=L^{2}(X, \mathcal{G}, \mu)$ and $P_{\mu}(f \mid \Re)=$ $E_{\mu}(f \mid \mathcal{G})$. Then it is easy to see that if $S$ is dominated, i.e., there is a measure $m$ on $\mathscr{F}$ with $\mu \ll m$ for all $\mu \in S$, then $\mathcal{G}$ is sufficient for $S$ if and only if $\mathscr{B}$ is weakly sufficient for $S$. 
EXAMPLE 1.10. Let $\Re$ be a von Neumann algebra acting on a Hilbert space $\mathcal{H}$ with a cyclic and separating vector $\Omega$ with $\|\Omega\|=1$, and $\varphi$ be a faithful normal state given by $\varphi(A)=\langle\Omega, A \Omega\rangle$. For each von Neumann subalgebra $\mathfrak{M}$ of $\mathfrak{R}$, let $S$ be the set of all states $\psi$ defined by $\psi(A)=$ $\langle T \Omega, A \Omega\rangle$ with $T \in \mathfrak{R}_{+}^{\prime}, T \Omega \in \overline{\mathfrak{M} \Omega}$ and $\left\|T^{1 / 2} \Omega\right\|=1$. Then it follows from Theorem 1.6 that $\mathfrak{M}$ is weakly sufficient for $S$. Furthermore Theorem 1.8 shows that $\mathfrak{M}$ is sufficient for $S$ if and only if there exists a conditional expectation $\varepsilon_{\varphi}$ of $\mathfrak{R}$ onto $\mathfrak{M}$ with $\varphi=\varphi \circ \varepsilon_{\varphi}$, which is if and only if $\mathfrak{M}$ is invariant under the modular automorphism group $\sigma_{t}^{\varphi}$ (cf. [29]).

2. Weak sufficiency of positive linear maps. In this section, let $\mathbb{Q}$ and $\Re$ be two *-algebras and $\gamma: \Re \rightarrow Q$ be a linear map such that $\gamma(I)=I, \gamma\left(B^{*}\right)=\gamma(B)^{*}$ and $\gamma(B)^{*} \gamma(B) \leq \gamma\left(B^{*} B\right)$ for all $B \in \Re$. We also assume that for every $B \in \Re$ there is a $c>0$ with $B^{*} B \leq c I$, which is satisfied if $\mathscr{B}$ is a $C^{*}$-algebra. Let $\delta_{\mathscr{Q}}$ and $\digamma_{\mathscr{B}}$ be the sets of all states of $Q$ and $\Re$. Then it is immediate that $\varphi \in \mathcal{S}_{\mathbb{Q}}$ implies $\varphi \circ \gamma \in \mathcal{S}_{\mathscr{B}}$. For each $\varphi \in \mathcal{S}_{\mathbb{Q}}$ and $A \in \mathbb{Q}$, define a linear functional $\varphi_{A}$ of $\mathbb{Q}$ by $\varphi_{A}\left(A_{1}\right)=$ $\varphi\left(A^{*} A_{1}\right)$. Then we have $\varphi_{A} \circ \gamma \prec \varphi \circ \gamma$ since

$$
\begin{aligned}
\mid\left(\varphi_{A} \circ \gamma\right) & \left(B B_{1}\right)|=| \varphi\left(A^{*} \gamma\left(B B_{1}\right)\right) \mid \\
& \leq \varphi\left(A^{*} A\right)^{1 / 2} \varphi\left(\gamma\left(B B_{1}\right)^{*} \gamma\left(B B_{1}\right)\right)^{1 / 2} \\
& \leq \varphi\left(A^{*} A\right)^{1 / 2} \varphi\left(\gamma\left(B_{1}^{*} B^{*} B B_{1}\right)\right)^{1 / 2} \\
& \leq \varphi\left(A^{*} A\right)^{1 / 2} c^{1 / 2}(\varphi \circ \gamma)\left(B_{1}^{*} B_{1}\right)^{1 / 2}
\end{aligned}
$$

for every $B, B_{1} \in \Re$ where $B^{*} B \leq c I$. Therefore $d\left(\varphi_{A} \circ \gamma\right) / d(\varphi \circ \gamma) \in$ $\pi_{\varphi \circ \gamma}(\Re)^{c}$ is defined.

Definition 2.1. We call $\gamma$ to be weakly sufficient for $S$ if for each $A \in \mathbb{Q}$ there exists a sequence $\left\{B_{n}\right\}$ in $\mathscr{B}$ such that

$$
\left[d\left(\varphi_{A} \circ \gamma\right) / d(\varphi \circ \gamma)\right] \Omega_{\varphi \circ \gamma}=s-\lim \pi_{\varphi \circ \gamma}\left(B_{n}\right) \Omega_{\varphi \circ \gamma}, \quad \varphi \in S .
$$

Definition 2.1 is compatible with Definition 1.5. Indeed we have

THEOREM 2.2. Let $\gamma: \Re \rightarrow @$ be a $*$-homomorphism. Then $\gamma$ is weakly sufficient for $S \subset \mathcal{S}_{\mathfrak{Q}}$ if and only if the *-subalgebra $\gamma \Re$ of $\mathbb{Q}$ is weakly sufficient for $S$.

Proof. If $\left\{\mathcal{H}_{\varphi}, \pi_{\varphi}, \Omega_{\varphi}\right\}$ is the cyclic representation of $\mathscr{Q}$ induced by $\varphi \in \mathcal{S}_{\mathscr{Q}}$, then the cyclic representation of $\Re$ induced by $\varphi \circ \gamma$ is obtained 
by $\left\{\overline{(\gamma \mathscr{B})_{\varphi}}, \pi_{\varphi} \circ \gamma, \Omega_{\varphi}\right\}$. Now it suffices to show that

$$
\left[d\left(\varphi_{A} \circ \gamma\right) / d(\varphi \circ \gamma)\right] \Omega_{\varphi}=P_{\varphi}(A \mid \gamma \mathscr{B}), \quad \varphi \in \mathcal{S}_{\mathscr{Q}}, A \in \mathbb{Q} .
$$

This follows from

$$
\begin{aligned}
\left\langle\left[d\left(\varphi_{A} \circ \gamma\right) / d(\varphi \circ \gamma)\right] \Omega_{\varphi}, \pi_{\varphi}(\gamma B) \Omega_{\varphi}\right\rangle=\left(\varphi_{A} \circ \gamma\right)(B) \\
=\varphi\left(A^{*}(\gamma B)\right)=\left\langle\pi_{\varphi}(A) \Omega_{\varphi}, \pi_{\varphi}(\gamma B) \Omega_{\varphi}\right\rangle \\
=\left\langle P_{\varphi}(A \mid \gamma \Re), \pi_{\varphi}(\gamma B) \Omega_{\varphi}\right\rangle, \quad B \in \mathscr{B} .
\end{aligned}
$$

We assume further that $\mathcal{Q}$ is abelian and $\gamma: \mathscr{B} \rightarrow \mathbb{Q}$ is completely positive, i.e.,

$$
\sum_{i, j=1}^{n} A_{i}^{*} \gamma\left(B_{i}^{*} B_{j}\right) A_{j} \geq 0
$$

for every $A_{1}, \ldots, A_{n} \in \mathbb{Q}$ and $B_{1}, \ldots, B_{n} \in \mathscr{B}$. Note (see [30, IV. 3]) that when $\mathbb{Q}$ and $\mathscr{B}$ are $C^{*}$-algebras, any completely positive map $\gamma: \mathscr{B} \rightarrow \mathbb{Q}$ with $\gamma(I)=I$ satisfies automatically $\gamma(B)^{*} \gamma(B) \leq \gamma\left(B^{*} B\right)$ for all $B \in \mathscr{B}$, and any positive linear map $\gamma: \mathscr{Q} \rightarrow \mathscr{Q}$ is completely positive if either $\mathscr{Q}$ or $\Re$ is abelian. Let $\mathscr{Q} \otimes \Re$ be the $*$-algebraic tensor product of $\mathscr{Q}$ and $\Re$. For each $\varphi \in \mathcal{S}_{\mathfrak{Q}}$, we can define the compound state $\varphi \otimes \gamma$ of $\mathscr{\Re} \otimes$ by

$$
(\varphi \otimes \gamma)(A \otimes B)=\left(\varphi_{A^{*}} \circ \gamma\right)(B)=\varphi(A(\gamma B)), \quad A \in \mathbb{Q}, B \in \Re,
$$

since

$$
(\varphi \otimes \gamma)\left(\left(\sum_{i=1}^{n} A_{i} \otimes B_{i}\right)^{*}\left(\sum_{i=1}^{n} A_{i} \otimes B_{i}\right)\right)=\varphi\left(\sum_{i, j=1}^{n} A_{i}^{*} \gamma\left(B_{i}^{*} B_{J}\right) A_{j}\right) \geq 0
$$

Identifying $Q$ and $\mathscr{B}$ with $*$-subalgebras $Q \otimes I$ and $I \otimes \mathscr{B}$ of $Q \otimes \Re$, we then have

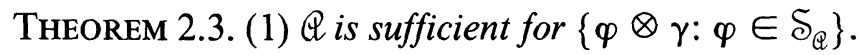

(2) $\gamma$ is weakly sufficient for $S \subset \mathcal{S}_{\mathfrak{Q}}$ if and only if $\mathscr{G}$ is weakly sufficient for $\{\varphi \otimes \gamma: \varphi \in S\}$.

Proof. (1) Define $\varepsilon: \mathbb{Q} \otimes \mathscr{B} \rightarrow \mathbb{Q}$ by $\varepsilon(A \otimes B)=A \gamma(B), A \in \mathbb{Q}, B \in \mathscr{B}$. Since $\gamma$ is completely positive and $Q$ is abelian, it follows that $\varepsilon$ is a conditional expectation of $\mathscr{Q} \otimes \mathscr{B}$ onto $\mathscr{Q}$. Hence (1) is seen from $(\varphi \otimes$ $\gamma) \circ \varepsilon=\varphi \otimes \gamma$ for all $\varphi \in \mathcal{S}_{\mathscr{Q}}$.

(2) For $\varphi \in \mathcal{S}_{\mathscr{Q}}$, let $\left\{\mathcal{K}_{\tilde{\varphi}}, \pi_{\tilde{\varphi}}, \Omega_{\tilde{\varphi}}\right\}$ be the cyclic representation of $\mathscr{Q} \otimes \Re$ induced by $\tilde{\varphi}=\varphi \otimes \gamma$. Since $\tilde{\varphi} \uparrow \Re=\varphi \circ \gamma$, the cyclic representation of $\mathscr{B}$ induced by $\varphi \circ \gamma$ is given by $\left\{\overline{\Re_{\tilde{\varphi}}}, \pi_{\tilde{\varphi}} \uparrow \Re, \Omega_{\tilde{\varphi}}\right\}$. Let $A \in \mathbb{Q}$, 
$B \in \Re$ and $T=d\left(\varphi_{A} \circ \gamma\right) / d(\varphi \circ \gamma) \in \pi_{\tilde{\varphi}}(\Re)^{c}$. It follows that

$$
\begin{aligned}
P_{\tilde{\varphi}}(A \otimes B \mid \Re) & =\left[d\left(\tilde{\varphi}_{A \otimes B} \uparrow \Re\right) / d(\tilde{\varphi}\lceil\Re)] \Omega_{\tilde{\varphi}}\right. \\
& =\left[d\left(\varphi_{A} \circ \gamma\right)_{B} / d(\varphi \circ \gamma)\right] \Omega_{\tilde{\varphi}}=T \pi_{\tilde{\varphi}}(B) \Omega_{\tilde{\varphi}},
\end{aligned}
$$

where the first equality is a special case of the equation in the proof of Theorem 2.2 and the last equality is seen from

$$
\begin{aligned}
& \left\langle\left[d\left(\varphi_{A} \circ \gamma\right)_{B} / d(\varphi \circ \gamma)\right] \Omega_{\tilde{\varphi}}, \pi_{\tilde{\varphi}}\left(B_{1}\right) \Omega_{\tilde{\varphi}}\right\rangle \\
& \quad=\left(\varphi_{A} \circ \gamma\right)\left(B^{*} B_{1}\right)=\left\langle T \pi_{\tilde{\varphi}}(B) \Omega_{\tilde{\varphi}}, \pi_{\tilde{\varphi}}\left(B_{1}\right) \Omega_{\tilde{\varphi}}\right\rangle, \quad B_{1} \in \mathscr{B} .
\end{aligned}
$$

The "if" part of (2) is now immediate by taking $B=I$. Conversely if $\gamma$ is weakly sufficient for $S$, then there exists a sequence $\left\{B_{n}\right\}$ in $\Re$ such that

$$
T \Omega_{\tilde{\varphi}}=s-\lim \pi_{\tilde{\varphi}}\left(B_{n}\right) \Omega_{\tilde{\varphi}}, \quad \varphi \in S .
$$

Since $\pi_{\tilde{\varphi}}(B)$ is bounded from $B^{*} B \leq c I$, we have

$$
P_{\tilde{\varphi}}(A \otimes B \mid \Re)=\pi_{\tilde{\varphi}}(B) T \Omega_{\tilde{\varphi}}=s-\lim \pi_{\tilde{\varphi}}\left(B B_{n}\right) \Omega_{\tilde{\varphi}}, \quad \varphi \in S .
$$

Hence $\mathscr{B}$ is weakly sufficient for $\{\varphi \otimes \gamma: \varphi \in S\}$.

EXAMPLE 2.4. Let $(X, \mathscr{F})$ and $(Y, \mathcal{G})$ be two measurable spaces and $\nu$ be a channel distribution from $(X, \mathscr{F})$ to $(Y, \mathcal{G})$, i.e., $\nu$ is a real-valued function on $X \times \mathcal{G}$ such that for every $x \in X, \nu(x, \cdot)$ is a probability measure on $\mathcal{G}$ and for every $B \in \mathcal{G}, \nu(\cdot, B)$ is $\mathscr{F}$-measurable on $X$. Let $\Re(X)$ and $\Re(Y)$ be the abelian $C^{*}$-algebras of bounded complex-valued measurable functions on $X$ and $Y$. Define a positive linear map $\gamma$ : $\Re(Y) \rightarrow \Re(X)$ by

$$
(\gamma g)(x)=\int_{Y} g(y) \nu(x, d y), \quad x \in X, g \in \mathscr{B}(Y) .
$$

Let $S$ be a set of probability measures on $\mathscr{F}$. For each $\mu \in S, \mu \otimes \gamma$ is given by

$$
(\mu \otimes \gamma)(f \otimes g)=\int_{X \times Y} f \otimes g d(\mu \otimes \nu), \quad f \in \mathscr{B}(X), g \in \mathscr{B}(Y),
$$

where $\mu \otimes \nu$ is the probability measure on $\mathscr{F} \otimes \mathcal{G}$ defined by $(\mu \otimes \nu) \times$ $(A \times B)=\int_{A} \nu(x, B) d \mu$. Then we see in connection with Theorem 2.3(2) that $\gamma$ is weakly sufficient for $S$ if and only if the $\sigma$-subalgebra $X \times \mathcal{G}=$ $\{X \times B: B \in \mathcal{G}\}$ of $\mathscr{F} \otimes \mathcal{G}$ is sufficient in the classical sense for $\{\mu \otimes \nu$ : $\mu \in S\}$. 
EXAMPLE 2.5. Let $\mathfrak{N}$ be a von Neumann algebra. An $\mathfrak{i}$-valued PO-measure $M$ on a measurable space $(X, \mathscr{F})$ is a map $M: \mathscr{F} \rightarrow \mathfrak{A}$ such that $M(F) \geq 0$ for all $F \in \mathscr{F}$ and $\sum_{n=1}^{\infty} M\left(F_{n}\right)=I$ ( $\sigma$-weakly) for every countable measurable partition $\left\{F_{n}\right\}$ of $X$. Let $\mathscr{G}(X)$ be the abelian $C^{*}$-algebra of bounded measurable functions on $X$. We define a positive linear map $\gamma: \mathscr{B}(X) \rightarrow \mathfrak{N}$ with $\gamma(1)=I$ by

$$
\varphi(\gamma(f))=\int_{X} f d(\varphi \circ M), \quad f \in \Re_{B}(X), \varphi \in \Re_{*} .
$$

For each $\varphi \in \mathbb{S}$, the cyclic representation $\left\{\mathcal{H}_{\varphi \circ \gamma}, \pi_{\varphi \circ \gamma}, \Omega_{\varphi \circ \gamma}\right\}$ of $\Re(X)$ induced by $\varphi \circ \gamma$ is given as follows $\mathcal{H}_{\varphi \circ \gamma}=L^{2}(X, \varphi \circ M), \pi_{\varphi \circ \gamma}(f)$ is the multiplication operator by $f$, and $\Omega_{\varphi \circ \gamma}=1$. For $A \in \mathfrak{R}$, $d\left(\varphi_{A} \circ \gamma\right) / d(\varphi \circ \gamma)$ is identical to the Radon-Nikodym derivative $d\left(\varphi_{A} \circ M\right) / d(\varphi \circ M)$ which is in $L^{2}(X, \varphi \circ M)$. Now assume that $\Re$ is $\sigma$-finite, so that $\mathfrak{R}$ has a faithful normal state. Then it is proved that $\gamma$ is weakly sufficient for $S \subset \subseteq$ if and only if for every $A \in \mathfrak{R}$ there exists a measurable function $f$ on $X$ satisfying

$$
d\left(\varphi_{A} \circ M\right) / d(\varphi \circ M)=f \quad \text { a.e. }[\varphi \circ M], \quad \varphi \in S .
$$

Further assume that $M$ is pure, i.e., $M$ is a spectral measure. Then $\gamma$ is a *-homomorphism and $\gamma(\mathscr{B}(X))$ is equal to the subalgebra $\mathfrak{M}=\{M(F)$ : $F \in \mathscr{F}\}^{\prime \prime}$. Hence Theorem 2.2 shows that $\gamma$ is weakly sufficient for $S$ if and only if $\mathfrak{M}$ is weakly sufficient for $S$.

EXAMPle 2.6. Let $\mathbb{Q}$ be a $C^{*}$-algebra and $C(\delta)$ be the abelian $C^{*}$-algebra of continuous functions on $\mathcal{S}$. Define a positive linear map $\gamma$ : $\mathbb{Q} \rightarrow C(\mathcal{S})$ with $\gamma(I)=1$ by $(\gamma A)(\omega)=\omega(A), A \in \mathbb{Q}, \omega \in \mathcal{S}$. For each $\rho \in \mathcal{S}$ and each abelian von Neumann subalgebra $\mathfrak{B}$ of $\pi_{\rho}(\mathscr{Q})^{\prime}$, we take the $\mathfrak{B}$-orthogonal measure $\lambda$ of $\rho$ (cf. [30,p. 241]). Now assume that $\mathbb{Q}$ is separable and $\mathfrak{B} \subset \mathcal{B}_{\rho}=\pi_{\rho}(\mathbb{Q})^{\prime \prime} \cap \pi_{\rho}(\mathbb{Q})^{\prime}$, i.e., $\lambda$ is a subcentral measure of $\rho$, and let $S$ be the set of all Borel probability measures $\mu$ on $\delta$ with $\mu \ll \lambda$. Then $\gamma$ is weakly sufficient for $S$. This is proved as follows. There is a $*$-isomorphism $\theta$ of $L^{\infty}(\delta, \lambda)$ onto $\mathfrak{B}$ such that

$$
\left\langle\Omega_{\rho}, \theta(f) \pi_{\rho}(A) \Omega_{\rho}\right\rangle=\int_{\S} f(\omega) \omega(A) d \lambda(\omega), \quad A \in \mathbb{Q}, f \in L^{\infty}(\Im, \lambda) .
$$

For each $\mu \in S$ and $f \in C(\delta)$, taking $g_{\mu n}=\min \left((d \mu / d \lambda)^{1 / 2}, n\right)$ we obtain 


$$
\begin{gathered}
(\mu \circ \gamma)(A)=\int_{\S} \omega(A) d \mu(\omega)=\lim \left\langle\theta\left(g_{\mu n}\right) \Omega_{\rho}, \pi_{\rho}(A) \theta\left(g_{\mu n}\right) \Omega_{\rho}\right\rangle, \\
\left(\mu_{f} \circ \gamma\right)(A)=\int_{\delta} \bar{f}(\omega) \omega(A) d \mu(\omega) \\
=\lim \left\langle\theta(f) \theta\left(g_{\mu n}\right) \Omega_{\rho}, \pi_{\rho}(A) \theta\left(g_{\mu n}\right) \Omega_{\rho}\right\rangle .
\end{gathered}
$$

Since $\left\{g_{\mu n}\right\}$ is Cauchy in $L^{2}(\mathcal{\delta}, \lambda)$, it follows that $\Phi_{\mu}=s-\lim \theta\left(g_{\mu n}\right) \Omega_{\rho}$ exists and

$$
\begin{gathered}
(\mu \circ \gamma)(A)=\left\langle\Phi_{\mu}, \pi_{\rho}(A) \Phi_{\mu}\right\rangle, \\
\left(\mu_{f} \circ \gamma\right)(A)=\left\langle\theta(f) \Phi_{\mu}, \pi_{\rho}(A) \Phi_{\mu}\right\rangle, \quad A \in \mathbb{Q} .
\end{gathered}
$$

Hence the cyclic representation of $\mathscr{Q}$ induced by $\mu \circ \gamma$ is given by

$$
\left\{\overline{\pi_{\rho}(\mathbb{Q}) \Phi_{\mu}}, \pi_{\rho}(\cdot) \uparrow \overline{\pi_{\rho}(\mathbb{Q}) \Phi_{\mu}}, \Phi_{\mu}\right\}
$$

and we have

$$
\left[d\left(\mu_{f} \circ \gamma\right) / d(\mu \circ \gamma)\right] \Phi_{\mu}=\theta(f) \Phi_{\mu} .
$$

Since $\mathcal{Q}$ is separable, there exists a sequence $\left\{A_{n}\right\}$ in $\mathbb{Q}$ such that $\pi_{\rho}\left(A_{n}\right) \rightarrow$ $\theta(f)$ (strongly), and hence

$$
\left[d\left(\mu_{f} \circ \gamma\right) / d(\mu \circ \gamma)\right] \Phi_{\mu}=s-\lim \pi_{\rho}\left(A_{n}\right) \Phi_{\mu}, \quad \mu \in S .
$$

This shows that $\gamma$ is weakly sufficient for $S$.

A linear map $\gamma: \Re \rightarrow \mathbb{Q}$ considered here describes more or less a quantum communication channel with the input space $\mathscr{Q}$ and the output space $\Re$ (cf. [15, 21]). Examples 2.4-2.6 provide classical-classical, quantum-classical and classical-quantum channels. Roughly speaking, the physical meaning of weak sufficiency of $\gamma$ is that the indirect measurement through $\gamma$ gives as much information (measured by the relative entropy) as the direct measurement of observables in $\mathbb{Q}$ given a set $S$ of input states (see $\S \S 3,4)$.

3. Relative entropy of states of $*$-algebras. We begin with the definitions of Araki's relative entropy and Uhlmann's relative entropy.

(I) Araki's relative entropy. Let $(\mathfrak{N}, \mathcal{H}, J, \mathcal{P})$ be a standard form of a von Neumann algebra $\Re$ (cf. $[2,12]$ ). Araki $[4,5]$ defined the relative entropy of normal positive linear functionals $\varphi$ and $\psi$ of $\mathfrak{N}$ as follows. 
There exist unique vector representatives $\Phi$ and $\Psi$ in $\mathscr{P}$ such that $\varphi(A)=$ $\langle\Phi, A \Phi\rangle$ and $\psi(A)=\langle\Psi, A \Psi\rangle$ for all $A \in \mathfrak{N}$. The operator $S_{\Psi, \Phi}$ with the domain

$$
D\left(S_{\Psi, \Phi}\right)=\mathfrak{R} \Phi+\left(I-s^{\mathfrak{R}^{\prime}}(\Phi)\right)
$$

is defined by

$$
S_{\Psi, \Phi}(A \Phi+\Omega)=s^{\mathfrak{N}}(\Phi) A^{*} \Psi, \quad A \in \mathfrak{R}, s^{\mathfrak{R}^{\prime}}(\Phi) \Omega=0,
$$

where $s^{\mathfrak{R}}(\Phi)$ denotes the $\mathfrak{R}$-support of $\Phi$. Then $S_{\Psi, \Phi}$ is a closable conjugate-linear operator and the relative modular operator $\Delta_{\Psi, \Phi}$ is defined by $\Delta_{\Psi, \Phi}=\left(S_{\Psi, \Phi}\right) * \overline{S_{\Psi, \Phi}}$. Let $\Delta_{\Psi, \Phi}=\int_{0}^{\infty} \lambda d e_{\Psi, \Phi}(\lambda)$ be the spectral decomposition of $\Delta_{\Psi, \Phi}$. The Araki's relative entropy $S(\psi \mid \varphi)$ is now given by

$$
S(\psi \mid \varphi)= \begin{cases}\int_{+0}^{\infty} \log \lambda d\left\langle\Psi, e_{\Psi, \Phi}(\lambda) \Psi\right\rangle & \text { if } \psi \ll \varphi \\ +\infty & \text { otherwise. }\end{cases}
$$

Note that the relative entropy $S(\psi \mid \varphi)$ is independent of the choice of a standard form of $\Re$ which is unique up to unitary equivalence. We used in [14] the notation $S(\varphi \mid \psi)$ instead of $S(\psi \mid \varphi)$.

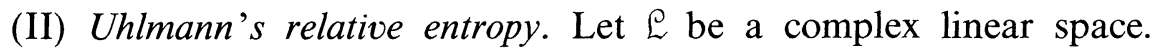
Given two seminorms $p$ and $q$ on $\mathcal{L}$, the quadratical mean $Q M(p, q)$ is defined by

$$
Q M(p, q)(x)=\sup _{\alpha \in H} \alpha(x, x)^{1 / 2}, \quad x \in \mathcal{L},
$$

where $H$ is the set of all positive hermitian forms $\alpha$ on $\_$satisfying $|\alpha(x, y)| \leq p(x) q(y)$ for all $x, y \in \mathcal{L}$. A function $t \mapsto p_{t}$ on $[0,1]$ whose values are seminorms on $\mathcal{L}$ is called a quadratical interpolation from $p$ to $q$ if for every $x \in \mathcal{L}$ the function $t \mapsto p_{t}(x)$ is continuous and if the following properties hold:

$$
\begin{array}{rlrl}
p_{t} & =Q M\left(p_{t_{1}}, p_{t_{2}}\right), & & t=\left(t_{1}+t_{2}\right) / 2, t_{1}, t_{2} \in[0,1], \\
p_{1 / 2} & =Q M(p, q), & & \\
p_{t / 2} & =Q M\left(p, p_{t}\right), \quad t \in[0,1], \\
p_{(1+t) / 2} & =Q M\left(q, p_{t}\right), \quad t \in[0,1] .
\end{array}
$$

Uhlmann [32] showed that for each positive hermitian forms $\alpha$ and $\beta$ there exists a unique function $t \mapsto Q F_{t}(\alpha, \beta)$ on $[0,1]$ with values in the set of 
positive hermitian forms on $\mathcal{E}$ such that the function $p_{t}$ given by $p_{t}(x)=$ $Q F_{t}(\alpha, \beta)(x, x)^{1 / 2}$ is the quadratical interpolation from $\alpha(x, x)^{1 / 2}$ to $\beta(x, x)^{1 / 2}$, and defined the relative entropy functional $S(\alpha ; \beta)(x)$ of $\alpha$ and $\beta$ by

$$
S(\alpha ; \beta)(x)=-\liminf _{t \rightarrow+0} \frac{1}{t}\left\{Q F_{t}(\alpha, \beta)(x, x)-\alpha(x, x)\right\}, \quad x \in \mathcal{L} .
$$

Now let $Q$ be a $*$-algebra, and $\varphi$ and $\psi$ be positive linear functionals of $Q$. The Uhlmann's relative entropy $S(\psi \mid \varphi)$ is defined by

$$
S(\psi \mid \varphi)=S\left(\psi^{R} ; \varphi^{L}\right)(I),
$$

where $\varphi^{L}$ and $\psi^{R}$ are the positive hermitian forms given by $\varphi^{L}(A, B)=$ $\varphi\left(A^{*} B\right)$ and $\psi^{R}(A, B)=\psi\left(B A^{*}\right)$.

For each normal positive linear functionals $\varphi$ and $\psi$ of a von Neumann algebra $\mathfrak{N}$, the Uhlmann's relative entropy is equal to the Araki's relative entropy. We here contain the proof for completeness.

Let $\mathcal{K}$ be the domain of $\left(I+\Delta_{\Psi, \Phi}\right)^{1 / 2}$, which becomes a Hilbert space with an inner product:

$$
\left(\Omega_{1}, \Omega_{2}\right)=\left\langle\left(I+\Delta_{\Psi, \Phi}\right)^{1 / 2} \Omega_{1},\left(I+\Delta_{\Psi, \Phi}\right)^{1 / 2} \Omega_{2}\right\rangle, \quad \Omega_{1}, \Omega_{2} \in \mathscr{K} .
$$

The operators $\left(I+\Delta_{\Psi, \Phi}\right)^{-1}$ and $\Delta_{\Psi, \Phi}\left(I+\Delta_{\Psi, \Phi}\right)^{-1}$ are positive bounded linear operators on $\mathcal{K}$. Define positive hermitian forms $\alpha$ and $\beta$ on $\mathcal{K}$ by

$$
\begin{aligned}
& \alpha\left(\Omega_{1}, \Omega_{2}\right)=\left(\Omega_{1}, \Delta_{\Psi, \Phi}\left(I+\Delta_{\Psi, \Phi}\right)^{-1} \Omega_{2}\right), \\
& \beta\left(\Omega_{1}, \Omega_{2}\right)=\left(\Omega_{1},\left(I+\Delta_{\Psi, \Phi}\right)^{-1} \Omega_{2}\right) .
\end{aligned}
$$

We then have (cf. [24], [32, Example 4])

$$
\begin{aligned}
Q F_{t}(\alpha, \beta)(\Omega, \Omega) & =\left(\Omega,\left[\Delta_{\Psi, \Phi}\left(I+\Delta_{\Psi, \Phi}\right)^{-1}\right]^{1-t}\left[\left(I+\Delta_{\Psi, \Phi}\right)^{-1}\right]^{t} \Omega\right) \\
& =\left\langle\Omega,\left(\Delta_{\Psi, \Phi}\right)^{1-t} \Omega\right\rangle, \quad t \in(0,1), \Omega \in \mathscr{K} .
\end{aligned}
$$

Since $\mathfrak{R} \Phi \subset \mathscr{K}$ and

$$
\begin{aligned}
& \psi^{R}(A, B)=\left(A \Phi, \Delta_{\Psi, \Phi}\left(I+\Delta_{\Psi, \Phi}\right)^{-1} B \Phi\right), \\
& \varphi^{L}(A, B)=\left(A \Phi,\left(I+\Delta_{\Psi, \Phi}\right)^{-1} B \Phi\right),
\end{aligned}
$$

it is easy to check that

$$
Q F_{t}\left(\psi^{R}, \varphi^{L}\right)(A, A)=Q F_{t}(\alpha, \beta)(A \Phi, A \Phi), \quad A \in \mathfrak{N} .
$$

Take the spectral decomposition $\Delta_{\Psi, \Phi}=\int_{0}^{\infty} \lambda d e_{\Psi, \Phi}(\lambda)$. If $\psi \ll \varphi$, then $\left(\Delta_{\Psi, \Phi}\right)^{1 / 2} \Phi=J S_{\Psi, \Phi} \Phi=J \Psi=\Psi$ and hence $\psi^{R}(I, I)=\left\|\left(\Delta_{\Psi, \Phi}\right)^{1 / 2} \Phi\right\|^{2}$. 
We have

$$
\begin{aligned}
S\left(\psi^{R} ; \varphi^{L}\right)(I) & =-\liminf _{t \rightarrow+0} \frac{1}{t}\left\{\left\langle\Phi,\left(\Delta_{\Psi, \Phi}\right)^{1-t} \Phi\right\rangle-\left\langle\Phi, \Delta_{\Psi, \Phi} \Phi\right\rangle\right\} \\
& =-\liminf _{t \rightarrow+0} \int_{+0}^{\infty} \lambda \frac{\lambda^{-t}-1}{t} d\left\langle\Phi, e_{\Psi, \Phi}(\lambda) \Phi\right\rangle \\
& =\int_{+0}^{\infty} \lambda \log \lambda d\left\langle\Phi, e_{\Psi, \Phi}(\lambda) \Phi\right\rangle \\
& =\int_{+0}^{\infty} \log \lambda d\left\langle\Psi, e_{\Psi, \Phi}(\lambda) \Psi\right\rangle,
\end{aligned}
$$

because the function $\left(\lambda^{-t}-1\right) / t$ converges decreasingly to $-\log \lambda$ as $t \rightarrow+0$. If $\psi \ll \varphi$ does not hold, then $\psi^{R}(I, I)<\left\|\left(\Delta_{\Psi, \Phi}\right)^{1 / 2} \Phi\right\|^{2}$ and hence $S\left(\psi^{R} ; \varphi^{L}\right)(I)=+\infty$. Thus the Uhlmann's relative entropy is equal to the Araki's one.

LEMMA 3.1. Let $Q$ be a $C^{*}$-algebra and $\pi$ be a nondegenerate representation of $\mathbb{Q}$ on a Hilbert space. If $\varphi$ and $\psi$ are positive linear functionals of $\mathbb{Q}$ having the normal extensions $\tilde{\varphi}$ and $\tilde{\psi}$ to $\pi(\mathbb{Q})^{\prime \prime}$ such that $\varphi(A)=\tilde{\varphi}(\pi(A))$ and $\psi(A)=\tilde{\psi}(\pi(A))$, then $S(\psi \mid \varphi)=S(\tilde{\psi} \mid \tilde{\varphi})$.

Proof. According to the Uhlmann's definition of relative entropy, it suffices to show that

$$
Q F_{t}\left(\psi^{R}, \varphi^{L}\right)(A, A)=Q F_{t}\left(\tilde{\psi}^{R}, \tilde{\varphi}^{L}\right)(\pi(A), \pi(A)), \quad t \in[0,1], A \in \mathbb{Q} .
$$

Let $\Gamma$ be the set of $t \in[0,1]$ for which the above equation holds for every $A \in \mathbb{Q}$. Let $H$ be the set of all positive hermitian forms $\alpha$ on $\mathbb{Q}$ satisfying

$$
\left|\alpha\left(A_{1}, A_{2}\right)\right| \leq \psi^{R}\left(A_{1}, A_{1}\right)^{1 / 2} \varphi^{L}\left(A_{2}, A_{2}\right)^{1 / 2}, \quad A_{1}, A_{2} \in \mathbb{Q},
$$

and $\tilde{H}$ be the set of all positive hermitian forms $\tilde{\alpha}$ on $\pi(\mathscr{Q})^{\prime \prime}$ satisfying

$$
\left|\tilde{\alpha}\left(Q_{1}, Q_{2}\right)\right| \leq \tilde{\psi}^{R}\left(Q_{1}, Q_{2}\right)^{1 / 2} \tilde{\varphi}^{L}\left(Q_{2}, Q_{2}\right)^{1 / 2}, \quad Q_{1}, Q_{2} \in \pi(\mathscr{Q})^{\prime \prime} .
$$

If $\tilde{\alpha} \in \tilde{H}$, then the form $\alpha$ on $\mathbb{Q}$ defined by $\alpha\left(A_{1}, A_{2}\right)=\tilde{\alpha}\left(\pi\left(A_{1}\right), \pi\left(A_{2}\right)\right)$ is in $H$. Conversely if $\alpha \in H$, then there exists a positive hermitian form $\hat{\alpha}$ on $\pi(\mathbb{Q})$ such that $\alpha\left(A_{1}, A_{2}\right)=\hat{\alpha}\left(\pi\left(A_{1}\right), \pi\left(A_{2}\right)\right)$ and hence

$$
\begin{aligned}
& \left|\hat{\alpha}\left(\pi\left(A_{1}\right), \pi\left(A_{2}\right)\right)\right| \\
& \quad \leq \tilde{\psi}^{R}\left(\pi\left(A_{1}\right), \pi\left(A_{2}\right)\right)^{1 / 2} \tilde{\varphi}^{L}\left(\pi\left(A_{2}\right), \pi\left(A_{2}\right)\right)^{1 / 2}, \quad A_{1}, A_{2} \in \mathbb{Q} .
\end{aligned}
$$


By the Kaplansky density theorem, $\hat{\alpha}$ can be uniquely extended to a positive hermitian form $\tilde{\alpha}$ on $\pi(\mathbb{Q})^{\prime \prime}$ which is in $\tilde{H}$. Therefore

$$
\begin{aligned}
Q F_{1 / 2}\left(\psi^{R}, \varphi^{L}\right)(A, A)= & \sup _{\alpha \in H} \alpha(A, A) \\
= & \sup ^{\tilde{\alpha}}(\pi(A), \pi(A)) \\
= & Q F_{1 / 2}\left(\tilde{\psi}^{R}, \tilde{\varphi}^{L}\right)(\pi(A), \pi(A)), \quad A \in \mathbb{Q} .
\end{aligned}
$$

This implies $1 / 2 \in \Gamma$. Noting that

$$
Q F_{t}\left(\psi^{R}, \varphi^{L}\right)(A, A) \leq \psi^{R}(A, A)^{1-t} \varphi^{L}(A, A)^{t}, \quad t \in[0,1], A \in \mathbb{Q},
$$

we can see by the similar arguments that $t \in \Gamma$ implies $t / 2 \in \Gamma$ and $(1+t) / 2 \in \Gamma$, and that $t_{1}, t_{2} \in \Gamma$ implies $\left(t_{1}+t_{2}\right) / 2 \in \Gamma$. Since $\Gamma$ is closed, we deduce that $\Gamma=[0,1]$.

In the above lemma, we can take as $\pi$ the cyclic representation induced by $\varphi+\psi$ or the universal representation of $\mathscr{Q}$.

We here remark that the relative entropy defined in (I) and (II) contains the usual relative entropies in the classical and quantum systems. Let $(X, \mathscr{F})$ be a measurable space, and $\mu$ and $\nu$ be probability measures on $\mathscr{F}$. Take a measure $m$ on $\mathscr{F}$ with $\mu, \nu \ll m$. Then $\mu$ and $\nu$ are naturally regarded as normal states of the abelian von Neumann algebra $\mathfrak{N}=$ $L^{\infty}(X, m)$ acting on $\mathcal{K}=L^{2}(X, m)$. Then the relative entropy $S(\nu \mid \mu)$ is equal to the classical relative entropy $I(\nu \mid \mu)$ (known as the Kullback-Leibler information):

$$
I(\nu \mid \mu)= \begin{cases}\int \frac{d \nu}{d \mu} \log \frac{d \nu}{d \mu} d \mu & \text { if } \nu \ll \mu \\ +\infty & \text { otherwise. }\end{cases}
$$

Indeed, $\Phi=(d \mu / d m)^{1 / 2}$ and $\Psi=(d \nu / d m)^{1 / 2}$ are vector representatives for $\mu$ and $\nu$, and $\Delta_{\Psi, \Phi}$ is the multiplication operator by $1_{\text {supp } \Phi}(\Psi / \Phi)^{2}$ where $1_{\text {supp } \Phi}$ is the characteristic function of the support of $\Phi$. If $\nu \ll \mu$, then we have

$$
\begin{aligned}
S(\nu \mid \mu) & =\int \Psi^{2} 1_{\text {supp } \Phi} \log (\Psi / \Phi)^{2} d m \\
& =\int \frac{d \nu}{d m}\left(\log \frac{d \nu}{d m}-\log \frac{d \mu}{d m}\right) d m=I(\nu \mid \mu) .
\end{aligned}
$$


Next let $\varphi$ and $\psi$ be normal states of the full von Neumann algebra $\mathfrak{N}=\mathbf{B}(\mathcal{H})$ on a Hilbert space $\mathcal{H}$. Then $\varphi$ and $\psi$ are given by $\varphi(A)=$ $\operatorname{Tr}\left(\rho_{\varphi} A\right)$ and $\psi(A)=\operatorname{Tr}\left(\rho_{\psi} A\right)$ with positive trace class operators $\rho_{\varphi}$ and $\rho_{\psi}$ on $\mathcal{H}$, and we obtain

$$
S(\psi \mid \varphi)=\operatorname{Tr}\left(\rho_{\psi} \log \rho_{\psi}-\rho_{\psi} \log \rho_{\varphi}\right) .
$$

The relative entropy $S(\psi \mid \varphi)$ has several basic properties such as joint convexity, monotonicity, lower semicontinuity, etc. (cf. $[4,5,32]$ ). The monotonicity is stated as follows (cf. [32, Proposition 18]). Let $\mathbb{Q}$ and $\Re$ be *-algebras and $\gamma: \mathscr{B} \rightarrow \mathbb{Q}$ be a linear map such that $\gamma(I)=I, \gamma\left(B^{*}\right)=$ $\gamma(B)^{*}$ and $\gamma(B)^{*} \gamma(B) \leq \gamma\left(B^{*} B\right)$ for all $B \in \Re$. If $\varphi$ and $\psi$ are positive linear functionals on $\mathcal{Q}$, then

$$
S(\psi \circ \gamma \mid \varphi \circ \gamma) \leq S(\psi \mid \varphi) .
$$

This monotonicity is applied to positive linear maps such as in Examples

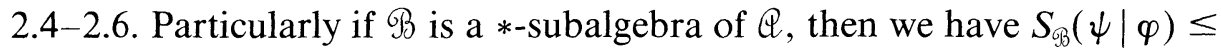
$S(\psi \mid \varphi)$ where $S_{\mathscr{G}}(\psi \mid \varphi)$ denotes the relative entropy of the restrictions $\varphi \uparrow \Re$ and $\psi \uparrow \Re$.

In connection with Example 2.6, it is proved that the relative entropy of states of a $C^{*}$-algebra is equal to that of their decomposition measures in some cases.

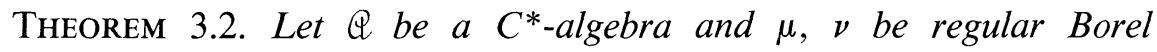
probability measures on $\mathcal{S}$ with barycenters $\varphi, \psi \in \mathcal{S}$. If there is a subcentral measure $\lambda$ on $\delta$ such that $\mu, \nu \ll \lambda$, then $S(\psi \mid \varphi)=I(\nu \mid \mu)$.

Proof. Let $\lambda$ be the $\mathfrak{B}$-orthogonal measure of $\rho \in \mathcal{S}$ with an abelian von Neumann subalgebra $\mathfrak{B}$ of $3_{\rho}=\pi_{\rho}(\mathbb{Q})^{\prime \prime} \cap \pi_{\rho}(\mathbb{Q})^{\prime}$, and $\theta$ be the *-isomorphism of $L^{\infty}(\mathcal{S}, \lambda)$ onto $\mathfrak{B}$ such that

$$
\left\langle\Omega_{\rho}, \theta(f) \pi_{\rho}(A) \Omega_{\rho}\right\rangle=\int_{\mathcal{S}} f(\omega) \omega(A) d \lambda(\omega), \quad A \in \mathbb{Q}, f \in L^{\infty}(\mathcal{\delta}, \lambda) .
$$

As is seen in Example 2.6, there exists a $\Phi_{\mu} \in \mathcal{H}_{\rho}$ such that $\varphi(A)=$ $\left\langle\Phi_{\mu}, \pi_{\rho}(A) \Phi_{\mu}\right\rangle$ for all $A \in \mathbb{Q}$. Hence $\varphi$ has the normal extension $\tilde{\varphi}$ to $\pi_{\rho}(Q)^{\prime \prime}$ and it is easily checked that

$$
\tilde{\varphi}(\theta(f))=\int_{\delta} f d \mu, \quad f \in L^{\infty}(\delta, \lambda) .
$$

Analogously $\psi$ has the normal extension $\tilde{\psi}$ to $\pi_{\rho}(Q)^{\prime \prime}$ satisfying

$$
\tilde{\psi}(\theta(f))=\int_{\delta} f d \nu, \quad f \in L^{\infty}(\delta, \lambda) .
$$


Using Lemma 3.1, we have

$$
S(\psi \mid \varphi)=S(\tilde{\psi} \mid \tilde{\varphi}) \geq S_{\mathfrak{B}}(\tilde{\psi} \mid \tilde{\varphi})=I(\nu \mid \mu) .
$$

The inverse inequality always holds by the monotonicity.

COROLlaRy 3.3. (1) Let $\varphi, \psi \in \mathcal{S}$ which satisfy the KMS condition with respect to a strongly continuous one-parameter automorphism group $\alpha_{t}$ of $Q$. If $\mu$ and $\nu$ are the central measures of $\varphi$ and $\psi$, then $S(\psi \mid \varphi)=I(\nu \mid \mu)$.

(2) Let $\{Q, G, \alpha\}$ be a $C^{*}$-dynamical system such that $\alpha_{G}$ is a large group of automorphisms of $\mathcal{Q}$, and $\varphi, \psi \in \mathcal{S}$ be $\alpha$-invariant. If $\mu$ and $\nu$ are the ergodic decomposition measures of $\varphi$ and $\psi$, then $S(\psi \mid \varphi)=I(\nu \mid \mu)$.

Proof. (1) Let $K$ be the set of all states satisfying the KMS condition with respect to $\alpha_{t}$. Then $K$ is a Choquet simplex and the central measure of $\rho \in K$ is identical to the unique maximal measure on $K$ representing $\rho$ (cf. [8, p. 121]). Hence it follows that $\lambda=(\mu+\nu) / 2$ is the central measure of $\rho=(\varphi+\psi) / 2$, so that Theorem 3.2 gives the desired equality.

(2) First note that the set $\delta_{\alpha}$ of all $\alpha$-invariant states becomes a Choquet simplex, because the condition of large group implies the $G$ abelianness (cf. [10]). Hence $\lambda=(\mu+\nu) / 2$ is the ergodic decomposition measure of $\rho=(\varphi+\psi) / 2$. It follows (cf. [26, Theorem 3.6], [27, Theorem 3.1]) that $\lambda$ is the $\mathfrak{B}$-orthogonal measure of $\rho$ with $\mathfrak{B}=\left(\pi_{\rho}(\mathbb{Q}) \cup U_{\rho}(G)\right)^{\prime}$ $=3_{\rho} \cap U_{\rho}(G)^{\prime}$ where $g \mapsto U_{\rho}(g)$ is the unitary representation of $G$ on $\mathcal{H}_{\rho}$ such that $\pi_{\rho}\left(\alpha_{g}(A)\right)=U_{\rho}(g) \pi_{\rho}(A) U_{\rho}(g)^{*}$ and $U_{\rho}(g) \Omega_{\rho}=\Omega_{\rho}$. Thus we have the desired equality.

4. Relative entropy, sufficiency and KMS condition. In this section, we establish some relations between the relative entropy, the sufficiency and the KMS condition in $W^{*}$-dynamical systems and $C^{*}$-dynamical systems. The following theorem is obvious from Definition 1.1 and the monotonicity of relative entropy.

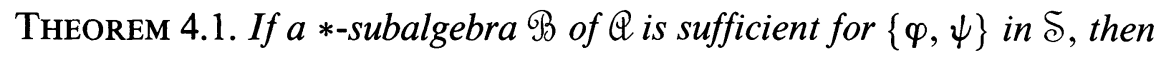
$S(\psi \mid \varphi)=S_{\mathfrak{B}}(\psi \mid \varphi)$.

TheOREM 4.2. Let $\mathfrak{N}$ be a von Neumann algebra and $\subseteq$ be the set of all normal states of $\mathfrak{R}$.

(1) Let $\{\mathfrak{N}, G, \alpha\}$ be a $W^{*}$-dynamical system. If $\varphi, \psi \in \subseteq$ are $\alpha$-invariant, then $S(\psi \mid \varphi)=S_{\mathfrak{R}^{\alpha}}(\psi \mid \varphi)$ where $\mathfrak{N}^{\alpha}$ is the fixed point subalgebra of $\alpha$. 
(2) Let $\alpha_{t}$ be a strongly continuous one-parameter automorphism group of $\mathfrak{R}$. If $\varphi, \psi \in \subseteq$ satisfy the $\mathrm{KMS}$ condition with respect to $\alpha_{t}$, then $S(\psi \mid \varphi)=S_{3}(\psi \mid \varphi)$ where $\mathbb{Z}=\mathfrak{R} \cap \mathfrak{R}^{\prime}$.

Proof. (1) Let $s(\varphi)$ and $s(\psi)$ be the support projections of $\varphi$ and $\psi$, which are in $\mathfrak{N}^{\alpha}$ from the $\alpha$-invariance of $\varphi$ and $\psi$. Since $S(\psi \mid \varphi)=$ $S_{\Re^{\alpha}}(\psi \mid \varphi)=+\infty$ if $s(\psi) \leq s(\varphi)$ does not hold, we assume that $s(\psi) \leq$ $s(\varphi)$. Letting $e=s(\varphi)$, we can define a $W^{*}$-dynamical system $\{\hat{\mathfrak{R}}, G, \hat{\alpha}\}$ by $\hat{\mathfrak{R}}=e \mathfrak{\Re} e$ and $\hat{\alpha}_{g}=\alpha_{g} \uparrow \hat{\mathfrak{R}}$. Then $\hat{\varphi}=\varphi \uparrow \hat{\mathfrak{R}}$ and $\hat{\psi}=\psi \uparrow \hat{\mathfrak{R}}$ are $\hat{\alpha}$-invariant. Since $\hat{\varphi}$ is faithful, it follows (see Example 1.4) that $\hat{\mathfrak{N}}^{\hat{\alpha}}=e \mathfrak{R}^{\alpha} e$ is sufficient for $\{\hat{\varphi}, \hat{\psi}\}$. Hence we have $S(\hat{\psi} \mid \hat{\varphi})=S_{e_{\Re^{\alpha}} e}(\hat{\psi} \mid \hat{\varphi})$ by Theorem 4.1. It now suffices to show the equations:

$$
S(\psi \mid \varphi)=S(\hat{\psi} \mid \hat{\varphi}) \quad \text { and } \quad S_{\Re^{\alpha}}(\psi \mid \varphi)=S_{\Re^{\alpha} e}(\hat{\psi} \mid \hat{\varphi}) .
$$

Define a linear map $\gamma: \mathfrak{R} \rightarrow \hat{\mathfrak{R}}$ by $\gamma(A)=e A e$. Then we have $\gamma(I)=e$, $\gamma\left(A^{*}\right)=\gamma(A)^{*}$ and $\gamma(A)^{*} \gamma(A) \leq \gamma\left(A^{*} A\right)$ for all $A \in \mathfrak{N}$. Since $\varphi=\hat{\varphi} \circ \gamma$ and $\psi=\hat{\psi} \circ \gamma$, the monotonicity gives $S(\psi \mid \varphi) \leq S(\hat{\psi} \mid \hat{\varphi})$. Next define a linear map $\hat{\gamma}: \hat{\mathfrak{R}} \rightarrow \mathfrak{N}$ by $\hat{\gamma}(B)=B+\hat{\varphi}(B)(I-e)$. Then we have $\hat{\gamma}(e)=$ $I, \hat{\gamma}\left(B^{*}\right)=\hat{\gamma}(B)^{*}$ and

$$
\begin{aligned}
\hat{\gamma}(B)^{*} \hat{\gamma}(B) & =B^{*} B+|\hat{\varphi}(B)|^{2}(I-e) \\
& \leq B^{*} B+\hat{\varphi}\left(B^{*} B\right)(I-e)=\hat{\gamma}\left(B^{*} B\right), \quad B \in \hat{\mathfrak{R}} .
\end{aligned}
$$

Since $\hat{\varphi}=\varphi \circ \hat{\gamma}$ and $\hat{\psi}=\psi \circ \hat{\gamma}$, the monotonicity again gives $S(\hat{\psi} \mid \hat{\varphi}) \leq$ $S(\psi \mid \varphi)$. We hence obtain the first equation and analogously the second equation.

(2) By the KMS condition, the support projections $s(\varphi)$ and $s(\psi)$ are in 3 (cf. [22, Lemma 5.1]). Letting $s(\psi) \leq s(\varphi)=e$, we define $\hat{\mathfrak{R}}=\mathfrak{\Re} e$ and $\hat{\alpha}_{t}=\alpha_{t} \uparrow \hat{\mathfrak{R}}$. Then $\hat{\varphi}=\varphi \uparrow \hat{\mathfrak{R}}$ and $\hat{\psi}=\psi \uparrow \hat{\mathfrak{R}}$ satisfy the KMS condition with respect to $\hat{\alpha}_{t}$. Since $\hat{\varphi}$ is faithful and hence $\hat{\alpha}_{t}=\sigma_{t}^{\hat{\varphi}}$ the modular automorphism group of $\hat{\varphi}$, it follows (see Example 1.2) that $\hat{3}=8 e$ is sufficient for $\{\hat{\varphi}, \hat{\psi}\}$. As in the proof of (1), we thus have

$$
S(\psi \mid \varphi)=S(\hat{\psi} \mid \hat{\varphi})=S_{3 e}(\hat{\psi} \mid \hat{\varphi})=S_{3}(\psi \mid \varphi) .
$$

THEOREM 4.3. Let $\alpha_{t}$ be a strongly continuous one-parameter automorphism group of $\mathfrak{N}$ and $\varphi, \psi \in \subseteq$. Assume that $\varphi$ satisfies the KMS condition with respect to $\alpha_{t}$.

(1) If $S(\psi \mid \varphi)=S_{\Re^{\alpha}}(\psi \mid \varphi)<+\infty$, then $\psi$ is $\alpha_{t}$-invariant.

(2) If $S(\psi \mid \varphi)=S_{3}(\psi \mid \varphi)<+\infty$, then $\psi$ satisfies the KMS condition with respect to $\alpha_{t}$. 
Proof. By the assumptions, we have $s(\varphi) \in 3 \cap \mathfrak{R}^{\alpha}$ and $s(\psi) \leq s(\varphi)$. As is seen from the proof of Theorem 4.2, we may suppose that $\varphi$ is faithful, so that $\alpha_{t}=\sigma_{t}^{\varphi}$ the modular automorphism group and $\mathfrak{R}^{\alpha}=Z_{\varphi}$ the centralizer of $\varphi$ (cf. [28, Lemma 15.8]). In [14, Corollaries 4.2 and 4.3], we proved (1) and (2) for the case when also $\psi$ is faithful. Now let $\psi$ be not faithful and $p=s(\psi)$.

(1) We first show that $p \in Z_{\varphi}$. Let $\hat{\mathfrak{R}}=\left(Z_{\varphi} \cup\{p\}\right)^{\prime \prime}, \hat{\varphi}=\varphi / \hat{\mathfrak{N}}$ and $\hat{\psi}=\psi \uparrow \hat{\mathfrak{R}}$. Then $\hat{\varphi}$ is a trace of $\hat{\mathfrak{R}}$ and we have $S(\hat{\psi} \mid \hat{\varphi})=S_{Z_{\mathrm{q}}}(\hat{\psi} \mid \hat{\varphi})$ $<+\infty$ by the assumption. Let $\varepsilon$ be the conditional expectation of $\hat{\Re}$ onto $Z_{\varphi}$ with $\hat{\varphi} \circ \varepsilon=\hat{\varphi}$. Define $\hat{\psi}^{\prime}=\hat{\psi} \circ \varepsilon, \hat{\psi}_{t}=(1-t) \hat{\psi}+t \hat{\varphi}$ and $\hat{\psi}_{t}^{\prime}=\hat{\psi}_{t} \circ \varepsilon$ $=(1-t) \hat{\psi}^{\prime}+t \hat{\varphi}$ for $0<t<1$. Since $\hat{\psi}_{t}$ is faithful, it follows by [14, Theorem 3.3] that

(*) $\quad\left\|\hat{\psi}_{t}^{\prime}-\hat{\psi}_{t}\right\| \leq\left\{2\left(S\left(\hat{\psi}_{t} \mid \hat{\varphi}\right)-S_{Z_{\varphi}}\left(\hat{\psi}_{t} \mid \hat{\varphi}\right)\right)\right\}^{1 / 2}, \quad 0<t<1$.

Since $\hat{\varphi}$ is a trace, there exists a positive self-adjoint operator $h$ affiliated with $\hat{\mathfrak{N}}$ such that $\hat{\psi}(A)=\hat{\varphi}(h A)$ for all $A \in \hat{\mathfrak{R}}$. Take the spectral decomposition $h=\int_{0}^{\infty} \lambda d e(\lambda)$. Noting that $\Delta_{\hat{\Psi}, \hat{\Phi}}=h$ and $\Delta_{\hat{\Psi}, \hat{\Phi}}=(1-t) h+t I$ where $\hat{\Phi}, \hat{\Psi}$, and $\hat{\Psi}_{t}$ are vector representatives of $\hat{\varphi}, \hat{\psi}$ and $\hat{\psi}_{t}$ in the standard form of $\hat{\mathfrak{R}}$, we have

$$
\begin{aligned}
& S(\hat{\psi} \mid \hat{\varphi})=\int_{0}^{\infty} \lambda \log \lambda d \hat{\varphi}(e(\lambda)) \\
& S\left(\hat{\psi}_{t} \mid \hat{\varphi}\right)=\int_{0}^{\infty}[(1-t) \lambda+t] \log [(1-t) \lambda+t] d \hat{\varphi}(e(\lambda)) .
\end{aligned}
$$

Since

$$
-\frac{1}{e} \leq[(1-t) \lambda+t] \log [(1-t) \lambda+t] \leq(1-t) \lambda \log \lambda
$$

it follows from the Lebesgue's convergence theorem that

$$
S(\hat{\psi} \mid \hat{\varphi})=\lim _{t \rightarrow+0} S\left(\hat{\psi}_{t} \mid \hat{\varphi}\right)
$$

and analogously

$$
S_{Z_{\varphi}}(\hat{\psi} \mid \hat{\varphi})=\lim _{t \rightarrow+0} S_{Z_{\varphi}}\left(\hat{\psi}_{t} \mid \hat{\varphi}\right)
$$

By letting $t \rightarrow+0$ in $(*)$, we obtain $\hat{\psi}^{\prime}=\hat{\psi}$, which implies that $Z_{\varphi}$ is sufficient for $\{\hat{\varphi}, \hat{\psi}\}$. Then it is easy to see that $h$ is affiliated with $Z_{\varphi}$, so that $p=s(h) \in Z_{\varphi}$. Now define a faithful state $\bar{\psi}=c \psi+(1-c) \bar{\varphi}$ where 
$c=\varphi(p)<1$ and $\bar{\varphi}=(1-c)^{-1} \varphi_{I-p}$. Since $s(\psi) \perp s(\bar{\varphi})$, by [5, Theorem 3.6] we have

$$
\begin{aligned}
S(\bar{\psi} \mid \varphi)= & c S(\psi \mid \varphi)+(1-c) S(\bar{\varphi} \mid \varphi) \\
& +c \log c+(1-c) \log (1-c), \\
S_{Z_{\varphi}}(\bar{\psi} \mid \varphi)= & c S_{Z_{\varphi}}(\psi \mid \varphi)+(1-c) S_{Z_{\varphi}}(\bar{\varphi} \mid \varphi) \\
& +c \log c+(1-c) \log (1-c) .
\end{aligned}
$$

Since $\bar{\varphi}$ is $\sigma_{t}^{\varphi}$-invariant and $\bar{\varphi} \leq(1-c)^{-1} \varphi$, it follows from Theorem 4.2 (1) that $S(\bar{\varphi} \mid \varphi)=S_{Z_{\varphi}}(\bar{\varphi} \mid \varphi)<+\infty$, and hence $S(\bar{\psi} \mid \varphi)=S_{Z_{\varphi}}(\bar{\psi} \mid \varphi)<$ $+\infty$. This implies by [14, Corollary 4.2] that $\bar{\psi}$ is $\sigma_{t}^{\varphi}$-invariant. Thus $\psi$ is $\sigma_{t}^{\varphi}$-invariant.

(2) Substituting $\underline{3}$ for $Z_{\varphi}$ in the proof of (1), we can show that $p \in Z$ and a faithful state $\bar{\psi}$ defined as above satisfies the KMS condition with respect to $\sigma_{t}^{\varphi}$, and thus $\psi$ satisfies the same condition.

Let $Q$ be a $C^{*}$-algebra and $\alpha_{t}$ be a strongly continuous one-parameter automorphism group of $\mathcal{Q}$. Let $\varphi \in \mathcal{S}$ and $\left\{\mathcal{H}_{\varphi}, \pi_{\varphi}, \Omega_{\varphi}\right\}$ be the cyclic representation of $\mathcal{Q}$ induced by $\varphi$. Suppose that $\varphi$ satisfies the KMS condition with respect to $\alpha_{t}$. Since $\varphi$ is $\alpha_{t}$-invariant, there is a strongly continuous one-parameter unitary group $U_{\varphi}(t)$ on $\mathcal{H}_{\varphi}$ such that $U_{\varphi}(t) \Omega_{\varphi}$ $=\Omega_{\varphi}$ and

$$
\pi_{\varphi}\left(\alpha_{t}(A)\right)=U_{\varphi}(t) \pi_{\varphi}(A) U_{\varphi}(t)^{*}, \quad t \in \mathbf{R}, A \in \mathbb{Q} .
$$

The normal extensions $\tilde{\varphi}$ and $\tilde{\alpha}_{t}$ of $\varphi$ and $\alpha_{t}$ to $\pi_{\varphi}(\mathscr{Q})^{\prime \prime}$ are given by

$$
\begin{array}{cl}
\tilde{\varphi}(Q)=\left\langle\Omega_{\varphi}, Q \Omega_{\varphi}\right\rangle, & Q \in \pi_{\varphi}(Q)^{\prime \prime}, \\
\tilde{\alpha}_{t}(Q)=U_{\varphi}(t) Q U_{\varphi}(t)^{*}, & t \in \mathbf{R}, Q \in \pi_{\varphi}(Q)^{\prime \prime},
\end{array}
$$

and it is known (cf. [1, Lemma 2.4]) that $\tilde{\varphi}$ satisfies the KMS condition with respect to $\tilde{\alpha}_{t}$, i.e., $\tilde{\alpha}_{t}=\sigma_{t}^{\tilde{\varphi}}$ the modular automorphism group of $\tilde{\varphi}$. Then we have

THEOREM 4.4. Let $Q, \alpha_{t}$ and $\varphi$ be as above. For each $\psi \in \mathcal{\delta}$ with $\psi \prec \varphi$, let $\tilde{\psi}$ be the normal extension of $\psi$ to $\pi_{\varphi}(Q)^{\prime \prime}$. Then the following conditions are equivalent:

(i) $\psi$ satisfies the KMS condition with respect to $\alpha_{t}$;

(ii) $3_{\varphi}=\pi_{\varphi}(\mathbb{Q})^{\prime \prime} \cap \pi_{\varphi}(\mathbb{Q})^{\prime}$ is sufficient for $\{\tilde{\varphi}, \tilde{\psi}\}$;

(iii) $3_{\varphi}$ is weakly sufficient for $\{\tilde{\varphi}, \tilde{\psi}\}$;

(iv) $(d \psi / d \varphi) \Omega_{\varphi} \in \overline{3_{\varphi} \Omega_{\varphi}}$; 
(v) $(D \tilde{\varphi}: D(\tilde{\varphi}+\tilde{\psi}))_{t} \in 3_{\varphi}$ for all $t \in \mathbf{R}$ where $(D \tilde{\varphi}: D(\tilde{\varphi}+\tilde{\psi}))_{t}$ is the Connes Radon-Nikodym derivative (cf. [9]);

(vi) $S(\psi \mid \varphi)=S_{3_{\varphi}}(\tilde{\psi} \mid \tilde{\varphi})$.

Proof. Note that $\tilde{\psi}$ is given by

$$
\tilde{\psi}(Q)=\left\langle(d \psi / d \varphi) \Omega_{\varphi}, Q \Omega_{\varphi}\right\rangle, \quad Q \in \pi_{\varphi}(\mathscr{Q})^{\prime \prime},
$$

and hence $\tilde{\psi} \prec \tilde{\varphi}$. Since there exists a conditional expectation $\varepsilon_{\tilde{\varphi}}$ of $\pi_{\varphi}(Q)^{\prime \prime}$ onto $3_{\varphi}$ with $\tilde{\varphi}=\tilde{\varphi} \circ \varepsilon_{\tilde{\varphi}}$, the equivalence of (ii), (iii) and (iv) follows from Theorem 1.6 (Remark) and the proof of Theorem 1.8. Because the KMS condition of $\psi$ with respect to $\alpha_{t}$ and the same of $\tilde{\psi}$ with respect to $\tilde{\alpha}_{t}$ are equivalent, it follows from [14, Theorem 2.3] that (i) and (ii) are equivalent. Since $(D \tilde{\varphi}: D(\tilde{\varphi}+\tilde{\psi}))_{t}=(D(\tilde{\varphi}+\tilde{\psi}): D \tilde{\varphi})_{t}^{*}$, we see by [14, Lemma 2.1] the equivalence of (ii) and (v). Finally the equivalence of (i) and (vi) follows from Theorems 4.2 (2) and 4.3 (2) and Lemma 3.1 if we prove $S_{\beta_{\varphi}}(\tilde{\psi} \mid \tilde{\varphi})<+\infty$. There exists a positive self-adjoint operator $h$ affiliated with $3_{\varphi}$ such that $\tilde{\psi}(Q)=\tilde{\varphi}(h Q)$ for all $Q \in 3_{\varphi}$. Take the spectral decomposition $h=\int_{0}^{\infty} \lambda d e(\lambda)$. Then the condition $\tilde{\psi} \prec \tilde{\varphi}$ gives rise to $\tilde{\varphi}\left(h^{2}\right)=\int_{0}^{\infty} \lambda^{2} d \tilde{\varphi}(e(\lambda))<+\infty$. Hence we have

$$
\begin{aligned}
S_{B_{\varphi}}(\tilde{\psi} \mid \tilde{\varphi}) & =\int_{0}^{\infty} \lambda \log \lambda d \tilde{\varphi}(e(\lambda)) \\
& \leq \int_{0}^{\infty} \lambda^{2} d \tilde{\varphi}(e(\lambda))<+\infty
\end{aligned}
$$

REMARK. Assuming only that $\psi$ has the normal extension $\tilde{\psi}$ to $\pi_{\varphi}(\Theta)^{\prime \prime}$ (which is necessarily a vector state), we obtain the equivalence of the conditions (i), (ii) and (v) in Theorem 4.4, which imply (vi) and are implied by the equality (vi) with a finite value. For the case of $\psi$ being dominated by $\varphi$, the condition (iv) can be replaced by $d \psi / d \varphi \in 3_{\varphi}$ (see e.g. [17, p. 104]). Also for the $\alpha_{t}$-invariance of $\psi \in \mathcal{S}$ with $\psi \prec \varphi$, we can obtain the similar equivalent conditions by substituting $Z_{\tilde{\varphi}}=\pi_{\varphi}(Q)^{\prime \prime} \cap$ $U_{\varphi}(\mathbf{R})^{\prime}$ for $3_{\varphi}$ in the above conditions (ii)-(vi).

Theorem 4.4 finds an application in quantum lattice systems. Let $L$ be a countable set and $\mathcal{H}_{0}$ a finite-dimensional Hilbert space. For each nonempty finite set $\Lambda \subset L$, let $\mathcal{H}_{\Lambda}=\otimes_{\mathrm{x} \in \Lambda} \mathcal{H}_{x}$ with $\mathcal{H}_{x}=\mathcal{H}_{0}$ and $\mathbb{Q}_{\Lambda}=$ $\mathbf{B}\left(\mathcal{K}_{\Lambda}\right)$. Then the quantum lattice system on $L$ is described by the quasi-local $C^{*}$-algebra $Q=\overline{U_{\Lambda \subset L} \mathbb{Q}_{\Lambda}}$. An interaction $\Phi$ is defined as a function from finite subsets $\Lambda \subset L$ into the self-adjoint elements of $\mathscr{Q}$ such that $\Phi(\Lambda) \in \mathbb{Q}_{\Lambda}$. Let $\varphi$ be a state of $\mathscr{Q}$ satisfying the Gibbs condition with 
respect to $\Phi$ (see [8] for the definition). Now assume that $\Phi$ satisfies

$$
\sum_{n=0}^{\infty} e^{r n}\left(\sup _{x \in L} \sum_{\substack{\Lambda \ni x \\|\Lambda|=n+1}}\|\Phi(\Lambda)\|\right)<+\infty
$$

for some $r>0$. Then the strongly continuous one-parameter automorphism group $\alpha_{t}^{\Phi}$ of $\mathcal{Q}$ can be given by

$$
\alpha_{t}^{\Phi}(A)=\lim _{\Lambda \rightarrow L} e^{i t H_{\Phi}(\Lambda)} A e^{-i t H_{\Phi}(\Lambda)}, \quad A \in \mathbb{Q}, t \in \mathbf{R},
$$

where $H_{\Phi}(\Lambda)=\Sigma_{X \subset \Lambda} \Phi(X)$. It is known (cf. [8,p. 268]) that $\psi \in \delta$ satisfies the Gibbs condition with respect to $\Phi$ if and only if $\psi$ satisfies the KMS condition with respect to $\alpha_{t}^{\Phi}$. Then we have

Corollary 4.5. Let $\mathbb{Q}, \Phi, \alpha_{t}=\alpha_{t}^{\Phi}$ and $\varphi$ be as above, and let $\psi \in \mathcal{S}$ with $\psi \prec \varphi$. Then the Gibbs condition for $\psi$ with respect to $\Phi$ is equivalent to each of the conditions (i)-(vi) in Theorem 4.4, and these conditions imply the following:

(vii) for each $\Lambda \subset L, \mathbb{Q}_{\Lambda^{c}}=\overline{\cup_{X \subset \Lambda^{c}} \mathbb{Q}_{X}}$ is weakly sufficient for $\{\varphi, \psi\}$;

(viii) for each $\Lambda \subset L, S(\psi \mid \varphi)=S_{\mathbb{Q}_{\Lambda} c}(\psi \mid \varphi)$.

Further if $\cup_{\Lambda \subset L} \pi_{\varphi}\left(Q_{\Lambda}\right) \Omega_{\varphi}$ is a core for the modular operator $\Delta_{\Omega_{\varphi}}$ associated with $\Omega_{\varphi}$, the condition (vii) conversely implies the Gibbs condition for $\psi$ with respect $\Phi$.

The main part of the corollary is immediate from Theorem 4.4 and the fact that $\mho_{\varphi}$ is identical to $\bigcap_{\Lambda \subset L} \pi_{\varphi}\left(Q_{\Lambda^{c}}\right)^{\prime \prime}$ the algebra of observables at infinity. The last part follows by [6, Lemma 3].

We finally give some notes on the translationally invariant case of $L=\mathbf{Z}^{d}$. Let $\tau$ be the automorphism group of translations on $\mathbf{Z}^{d}$. Let $\Phi$ be a $\tau$-invariant interaction satisfying $\Sigma_{\Lambda \ni 0} e^{r|\Lambda|}\|\Phi(\Lambda)\|<+\infty$ for some $r>0$. A $\tau$-invariant state $\varphi$ is said to be equilibrium with respect to $\Phi$ if the following variational equality holds (see $[17,25]): P(\Phi)=s(\varphi)-$ $\varphi\left(A_{\Phi}\right)$ where $s(\varphi)$ is the mean entropy of $\varphi$ and

$$
\begin{aligned}
P(\Phi) & =\lim _{\substack{\Lambda \rightarrow \infty \\
\text { (van Hove) }}}|\Lambda|^{-1} \log \operatorname{tr}_{\Lambda}\left(e^{-H_{\Phi}(\Lambda)}\right), \\
A_{\Phi} & =\sum_{\Lambda \ni 0}|\Lambda|^{-1} \Phi(\Lambda) .
\end{aligned}
$$

Then the equilibrium condition with respect to $\Phi$, the Gibbs condition with respect to $\Phi$ and the KMS condition with respect to $\alpha_{t}^{\Phi}$ are all equivalent for $\tau$-invariant states of $\mathscr{Q}$ (cf. $[3,8,20])$. Let $\varphi, \psi \in \mathcal{S}$ be 
$\tau$-invariant. Since $\left\{\mathscr{Q}, \mathbf{Z}^{\mathrm{d}}, \tau\right\}$ is asymptotically abelian, we obtain $S(\psi \mid \varphi)$ $=I(\nu \mid \mu)$ by Corollary $3.3(2)$ where $\mu$ and $\nu$ are the ergodic decomposition measures of $\varphi$ and $\psi$. If $\varphi$ is equilibrium and $\psi \prec \varphi$ (or more weakly $\psi$ has the normal extension to $\left.\pi_{\varphi}(Q)^{\prime \prime}\right)$, then it can be proved that $\nu \ll \mu$, so that $\psi$ is automatically equilibrium because $\mu$ is supported on the set of equilibrium states.

\section{REFERENCES}

1. H. Araki, Multiple time analyticity of a quantum statistical state satisfying the KMS boundary condition, Publ. RIMS, Kyoto Univ. Ser. A, 4 (1968), 361-371.

2 . __ Some properties of modular conjugation operator of von Neumann algebras and a non-commutative Radon-Nikodym theorem with a chain rule, Pacific J. Math., 50 (1974), 309-354.

3. On the equivalence of the KMS condition and the variational principle for quantum lattice systems, Comm. Math. Phys., 38 (1974), 1-10.

$4 . \quad$, Relative entropy of states of von Neumann algebras, Publ. RIMS, Kyoto Univ., 11 (1976), 809-833.

$5 . \quad$, Relative entropy for states of von Neumann algebras II, Publ. RIMS, Kyoto Univ., 13 (1977), 173-192.

6. H. Araki and A. Kishimoto, On clustering property, Rep. Math. Phys., 10 (1976), 275-281.

7. R. R. Bahadur, Sufficiency and statistical decision functions, Ann. Math. Statist., 25 (1954), 423-462.

8. O. Bratteli and D. W. Robinson, Operator Algebras and Quantum Statistical Mechanics II, Springer, New York, 1981.

9. A. Connes, Une classification des facteurs de type III, Ann. Sci. École Norm. Sup. Sér. 4,6 (1973), 133-252.

10. S. Doplicher, D. Kastler and E. Størmer, Invariant states and asymptotic abelianness, J. Funct. Anal., 3 (1969), 419-434.

11. S. P. Gudder and R. L. Hudson, A noncommutative probability theory, Trans. Amer. Math. Soc., 245 (1978), 1-41.

12. U. Haagerup, The standard form of von Neumann algebras, Math. Scand., 37 (1975), 271-283.

13. P. R. Halmos and L. J. Savage, Application of the Radon-Nikodym theorem to the theory of sufficient statistics, Ann. Math. Statist., 20 (1949), 225-241.

14. F. Hiai, M. Ohya and M. Tsukada, Sufficiency, KMS condition and relative entropy in von Neumann algebras, Pacific J. Math., 96 (1981), 99-109.

15. A. S. Holevo, Problems in the mathematical theory of quantum communication channels, Rep. Math. Phys., 12 (1977), 273-278.

16. _ Investigations in the General Theory of Statistical Decisions, Amer. Math. Soc., Proc. Steklov Institute of Math., no. 124, 1978.

17. R. B. Israel, Convexity in the Theory of Lattice Gases, Princeton Univ. Press, Princeton, 1979.

18. I. Kovács and J. Szücs, Ergodic type theorems in von Neumann algebras, Acta Sci. Math., 27 (1966), 233-246.

19. S. Kullback and R. A. Leibler, On information and sufficiency, Ann. Math. Statist., 22 (1951), 79-86.

20. O. E. Lanford III and D. W. Robinson, Statistical mechanics of quantum spin systems. III, Comm. Math. Phys., 9 (1968), 327-338. 
21. M. Ohya, Quantum ergodic channels in operator algebras, J. Math. Anal. Appl., 84 (1981), 318-327.

22. G. K. Pedersen and M. Takesaki, The Radon-Nikodym theorems for von Neumann algebras, Acta Math., 130 (1973), 53-87.

23. R. T. Powers, Self-adjoint algebras of unbounded operators, Comm. Math. Phys., 21 (1971), 85-124.

24. W. Pusz and S. L. Woronowicz, Functional calculus for sesquilinear forms and the purification map, Rep. Math. Phys., 8 (1975), 159-170.

25. D. Ruelle, Statistical Mechanics: Rigorous Results, Benjamin, New York-Amsterdam, 1969.

26. , Integral representation of states on a $C^{*}$-algebra, J. Functional Anal., 6 (1970), $116-151$.

27. E. Størmer, Large groups of automorphisms of $C^{*}$-algebras, Comm. Math. Phys., 5 (1967), 1-22.

28. M. Takesaki, Tomita's Theory of Modular Hilbert Algebras and its Applications, Springer, Lecture notes in math., Vol. 128, 1970.

$29 . \quad$ C Conditional expectations in von Neumann algebras, J. Functional Anal., 9 (1972), 306-321.

30. Theory of Operator Algebras I, Springer, New York, 1979.

31. J. Tomiyama, On the projection of norm one in $W^{*}$-algebras, Proc. Japan Acad., 33 (1957), 608-612.

32. A. Uhlmann, Relative entropy and the Wigner-Yanase-Dyson-Lieb concavity in an interpolation theory, Comm. Math. Phys., 54 (1977), 21-32.

33. H. Umegaki, Conditional expectation in an operator algebra, III, Kōdai Math. Sem. Rep., 11 (1959), 51-64.

34. Conditional expectation in an operator algebra, IV (entropy and information), Kōdai Math. Sem. Rep., 14 (1962), 59-85.

Received July 6, 1981 and in revised form November 11, 1981.

SCIENCE UNIVERSITY OF TOKYO

NodA City, Chiba 278, JAPAN 


\section{PACIFIC JOURNAL OF MATHEMATICS}

\section{EDITORS}

Donald BabbitT (Managing Editor)

University of California

Los Angeles, CA 90024

Hugo Rossi

University of Utah

Salt Lake City, UT 84112

C. C. Moore and Arthur Ogus

University of California

Berkeley, CA 94720
J. DugunduI

Department of Mathematics

University of Southern California

Los Angeles, CA 90089-1113

R. FinN and H. SAmelson

Stanford University

Stanford, CA 94305

\section{ASSOCIATE EDITORS}
R. ARENS
E. F. BECKENBACH
B. H. NEUMANN
F. WOLF
K. YosHIDA (1906-1982)

\section{SUPPORTING INSTITUTIONS}

UNIVERSITY OF ARIZONA

UNIVERSITY OF BRITISH COLUMBIA

CALIFORNIA INSTITUTE OF TECHNOLOGY

UNIVERSITY OF CALIFORNIA

MONTANA STATE UNIVERSITY

UNIVERSITY OF NEVADA, RENO

NEW MEXICO STATE UNIVERSITY

OREGON STATE UNIVERSITY
UNIVERSITY OF OREGON

UNIVERSITY OF SOUTHERN CALIFORNIA

STANFORD UNIVERSITY

UNIVERSITY OF HAWAII

UNIVERSITY OF TOKYO

UNIVERSITY OF UTAH

WASHINGTON STATE UNIVERSITY

UNIVERSITY OF WASHINGTON 


\section{Pacific Journal of Mathematics}

Vol. 107, No. $1 \quad$ January, 1983

John Kelly Beem and Phillip E. Parker, Klein-Gordon solvability and the

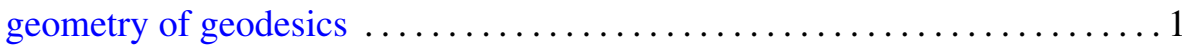

David Borwein and Amnon Jakimovski, Transformations of certain

sequences of random variables by generalized Hausdorff matrices ..... 15

Willy Brandal and Erol Barbut, Localizations of torsion theories . . . . . . . 227

John David Brillhart, Paul Erdős and Richard Patrick Morton, On sums

of Rudin-Shapiro coefficients. II ........................... 39

Martin Lloyd Brown, A note on tamely ramified extensions of rings $\ldots \ldots \ldots 71$

Chang P'ao Ch'ên, A generalization of the Gleason-Kahane-Żelazko

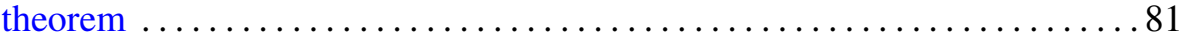

I. P. de Guzman, Annihilator alternative algebras $\ldots \ldots \ldots \ldots \ldots \ldots$. . . . 89

Ralph Jay De Laubenfels, Extensions of $d / d x$ that generate uniformly

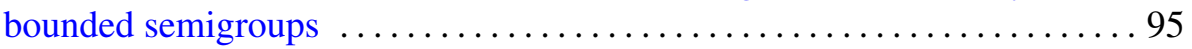

Patrick Ronald Halpin, Some Poincaré series related to identities of $2 \times 2$

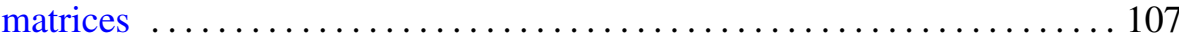

Fumio Hiai, Masanori Ohya and Makoto Tsukada, Sufficiency and

relative entropy in $*$-algebras with applications in quantum systems . . . 117

Dean Robert Hickerson, Splittings of finite groups $\ldots \ldots \ldots \ldots \ldots \ldots \ldots 14$

Jon Lee Johnson, Integral closure and generalized transforms in graded

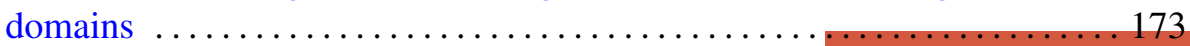

Maria Grazia Marinari, Francesco Odetti and Mario Raimondo, Affine

curves over an algebraically nonclosed field . ................. 179

Douglas Shelby Meadows, Explicit PL self-knottings and the structure of

PL homotopy complex projective spaces $\ldots \ldots \ldots \ldots \ldots \ldots \ldots \ldots \ldots$

Charles Kimbrough Megibben, III, Crawley's problem on the unique

$\omega$-elongation of $p$-groups is undecidable .................... 205

Mary Elizabeth Schaps, Versal determinantal deformations $\ldots \ldots \ldots \ldots 213$

Stephen Scheinberg, Gauthier's localization theorem on meromorphic

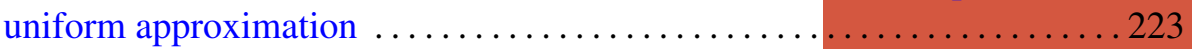

Peter Frederick Stiller, On the uniformization of certain curves . . . . . . . 229

Ernest Lester Stitzinger, Engel's theorem for a class of algebras . . . . . . . . 245

Emery Thomas, On the zeta function for function fields over $F_{p} \ldots \ldots \ldots 251$ 